Images du travail, travail des images

\title{
Images et relations en maison de retraite
}

Images and Relationships in Retirement Homes

Delphine Moras

\section{CpenEdition}

Journals

Édition électronique

URL : http://journals.openedition.org/itti/946

DOI : $10.4000 /$ itti.946

\section{Éditeur}

Université de Poitiers

\section{Référence électronique}

Delphine Moras, «Images et relations en maison de retraite », Images du travail, travail des images [En ligne], 4 | 2017, mis en ligne le 01 septembre 2017, consulté le 14 avril 2021. URL : http:// journals.openedition.org/itti/946 ; DOI : https://doi.org/10.4000/itti.946

Ce document a été généré automatiquement le 14 avril 2021.

Images du travail, travail des images 


\title{
Images et relations en maison de retraite
}

\author{
Images and Relationships in Retirement Homes
}

Delphine Moras

1 L'article s'appuie sur une expérience photographique tirée d'une démarche ethnographique dans le cadre d'une recherche doctorale. L'objectif ici est de présenter la photographie comme un cadre d'enquête où le sujet de la recherche se livre en images. La pratique photographique devient une manière de faire du terrain pour décrypter les interfaces entre soignants et soignés et les images produites révèlent des données que l'évidence du quotidien écarte de l'analyse. La présence de l'appareil entraine des postures et des interactions nouvelles riches en données ethnographiques.

2 La dimension épistémologique d'une recherche interroge ce rapport entre méthode utilisée et objet d'étude. Ici, l'usage de la photographie fait partie intégrante de l'apperception du soin. Comment la pratique de la photographie amène-t-elle un cadre postural favorable à la compréhension de la relation particulière soignant-soigné ? Qu'apporte ce rapport qui se crée derrière l'objectif de l'appareil photographique?

3 L'expérience s'est déroulée sur une semaine dans un établissement lyonnais qui accueille 75 personnes, après dix mois d'observation par immersion. Si au début ma présence avec l'appareil se cantonnait aux lieux communs, au quatrième jour j'ai été invitée par le personnel avec l'accord des personnes résidentes à entrer dans les chambres et assister à des temps de soins tels que le massage, les changements de protection, les toilettes, ou encore les séances de balnéothérapie. Sur les 851 photos réalisées, 217 ont été mises de côté pour leur caractère flou et indistinct et les 634 restantes présentaient un ensemble de peaux et de chairs qu'il fallait trier et analyser. Un travail de discernement a été nécessaire afin que ce corpus soit utilisable. Le noir et blanc a été privilégié pour faire ressortir la texture de la peau, également pour rompre avec la polychromie de la vie courante ${ }^{1}$.

4 Partant des gestes et postures, deux catégories significatives dans la situation de soin, une typographie relationnelle apparait entre une technique photographique et une 
gestualité soignante, pour finir sur un retour méthodologique qui permet d'interroger la pertinence de cette méthode dans le cadre d'une recherche sur le soin en établissement gérontologique.

\section{Les gestes et les postures}

En 1977, Grégory Bateson s'associait aux entretiens de Marguerite Mead pour rendre compte aux travers les gestes photographiés la dimension sociale de la communauté balinaise (Bateson, 1977). Cependant les études photographiques des gestes restent dans le domaine du langage quotidien. Desmond Morris consacre un ouvrage sur la main dans le monde occidental (Desmond, 1979) et place le corps au sein des rapports avec l'environnement (Desmond, 1985). La main, dans le cadre professionnel, exprime une transmission de savoirs faire mais la gestualité semble s'objectiver pour saisir globalement la construction d'une pratique.

Dans le soin, la main se fait le prolongement d'une relation animée par les actes. Le corps est un objet sensible et les approches photographiques analysées par Pierre Jérôme Jehel (2000) montrent comment la pratique s'inscrit face à un sujet. Le positionnement photographique construit des rapports qui s'engagent avec le domaine étudié en les relevant.

\subsection{Gestes et postures significatives}

7 La photographie a été l'occasion de découvrir une multitude de petits gestes. Si l'action et la mobilité dirigent d'emblée le regard du visiteur d'une maison de retraite vers la vivacité soignante, l'objectif photographique s'est arrêté sur des mouvements plus lents et moins amples, réalisés par les résident(e)s. Le frottement le long d'une canne, le froissement d'un mouchoir, ainsi que les mouvements des doigts les uns avec les autres, marquent une activité dense et dynamique. L'objectif de l'appareil, en observant ces spécificités, ne se posait pas en mandataire mais se souhaitait familier. 
Figure 1. De petits mouvements de doigts

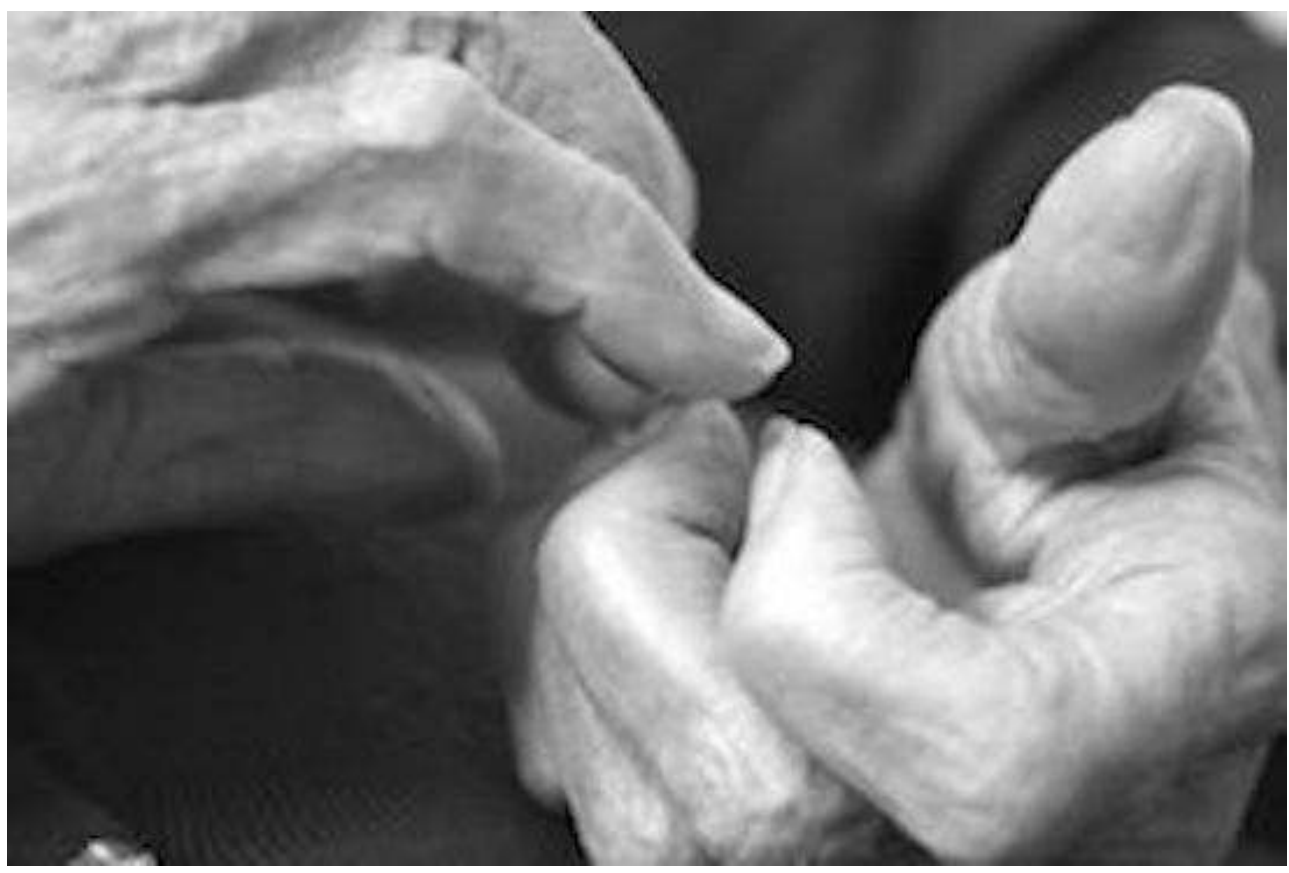

Mme P, atteinte de la maladie d'Alzheimer au stade sévère, fait souvent de petits gestes, elle semble jouer avec ses doigts. Toujours en mouvement, son comportement laisse penser à une forte activité introspective. Pensait-elle? Se créait-elle des espaces de souvenirs ?*

*Le cadrage serré accompagnait cette proximité recherchée. Ainsi, l'arrêt sur les détails invite l'observateur à dépasser son premier regard et à distinguer des gestes plus caractéristiques et significatifs. Derrière l'appareil, je découvrais les troubles praxiques et les difficultés particulières que le corps de ces personnes âgées vivait et que les bras des soignants accueillaient.

9 Mme L atteinte de la maladie d'Alzheimer se tient au bord du lit, elle hésite à se lever pour aller dans la salle de bain. Malgré l'insistance de Marie, l'aide soignante de l'unité protégée, elle se retient au lit, elle s'agrippe fortement. Il faudra un moment à Marie pour arriver à lui faire lâcher la barre du lit médicalisé. 
Figure 2. Main sur le barreau du lit

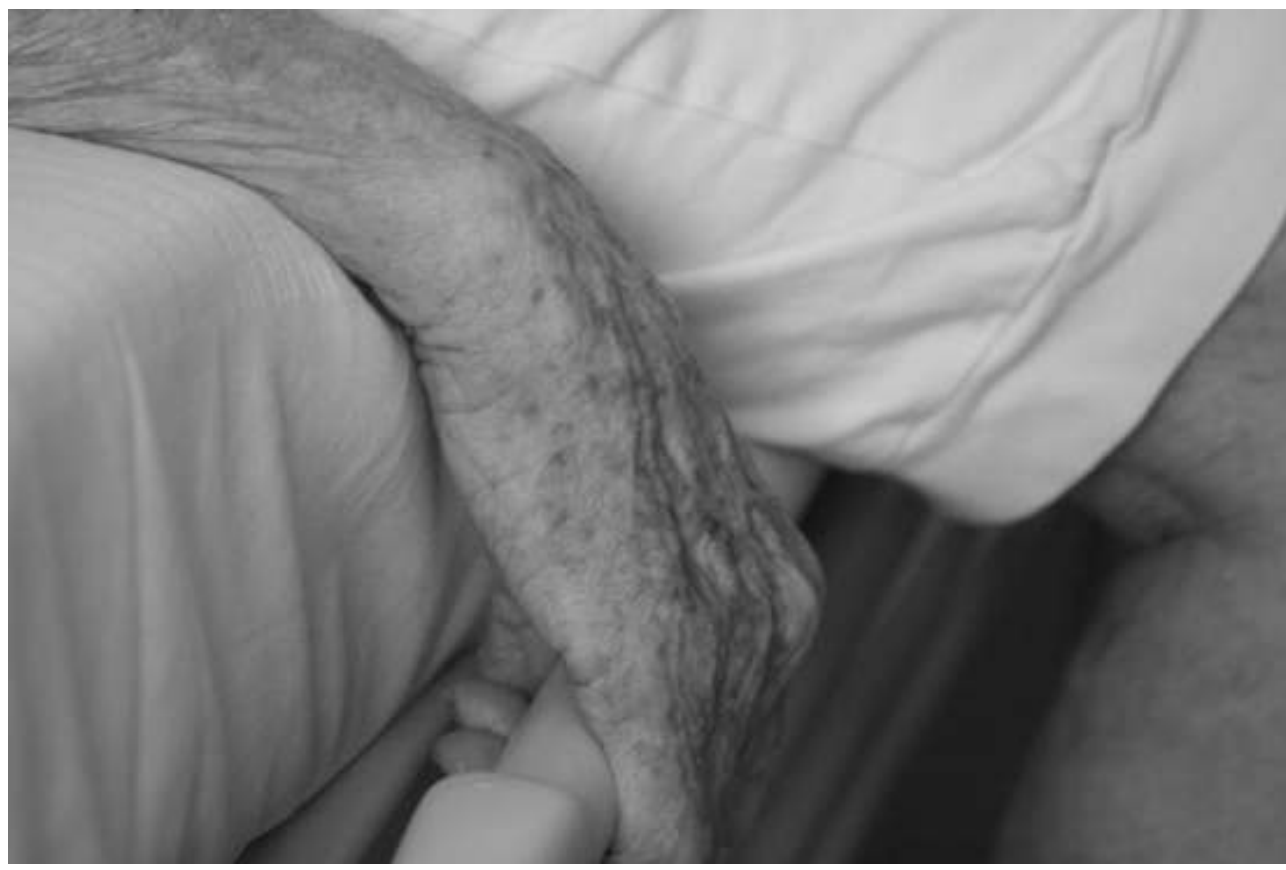

Le grasping est un geste significatif chez les personnes atteintes de la maladie d'Alzheimer. En référence au réflexe primaire du nouveau-né, cela consiste à fermer les doigts sur un objet placé dans la paume de la main. Ce geste d'agrippement caractérise une appréhension et une peur viscérale et instinctive qui fait partie des réactions courantes dans l'accompagnement. Ce saisissement extrême et prolongé entraine des méfiances et des craintes.

De plus, attendre en maison de retraite est une attitude fréquente mais polymorphique dans son usage particulier de la gestion du temps et de l'espace.

Figure 3. Mme $\mathrm{J}$ attend dans le jardin le début de l'animation musicale

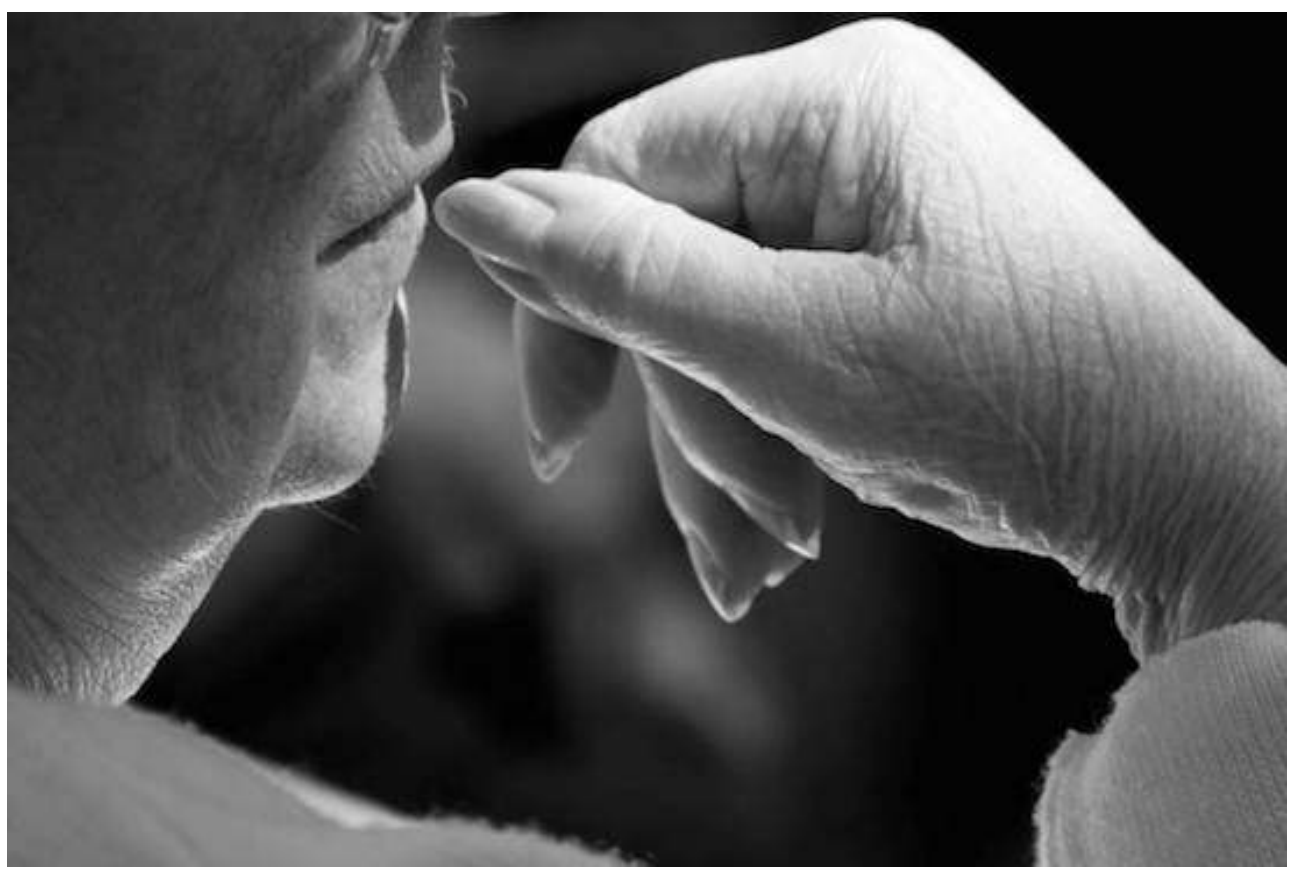

Mme J, par cet après-midi de mai, prend le soleil. Ce mercredi-là, une animation musicale était donnée dans le parc de la résidence, assise parmi d'autres personnes, elle attendait le début de l'activité. 
Figure 4. Attentes et pensées de Mme $\mathrm{K}$

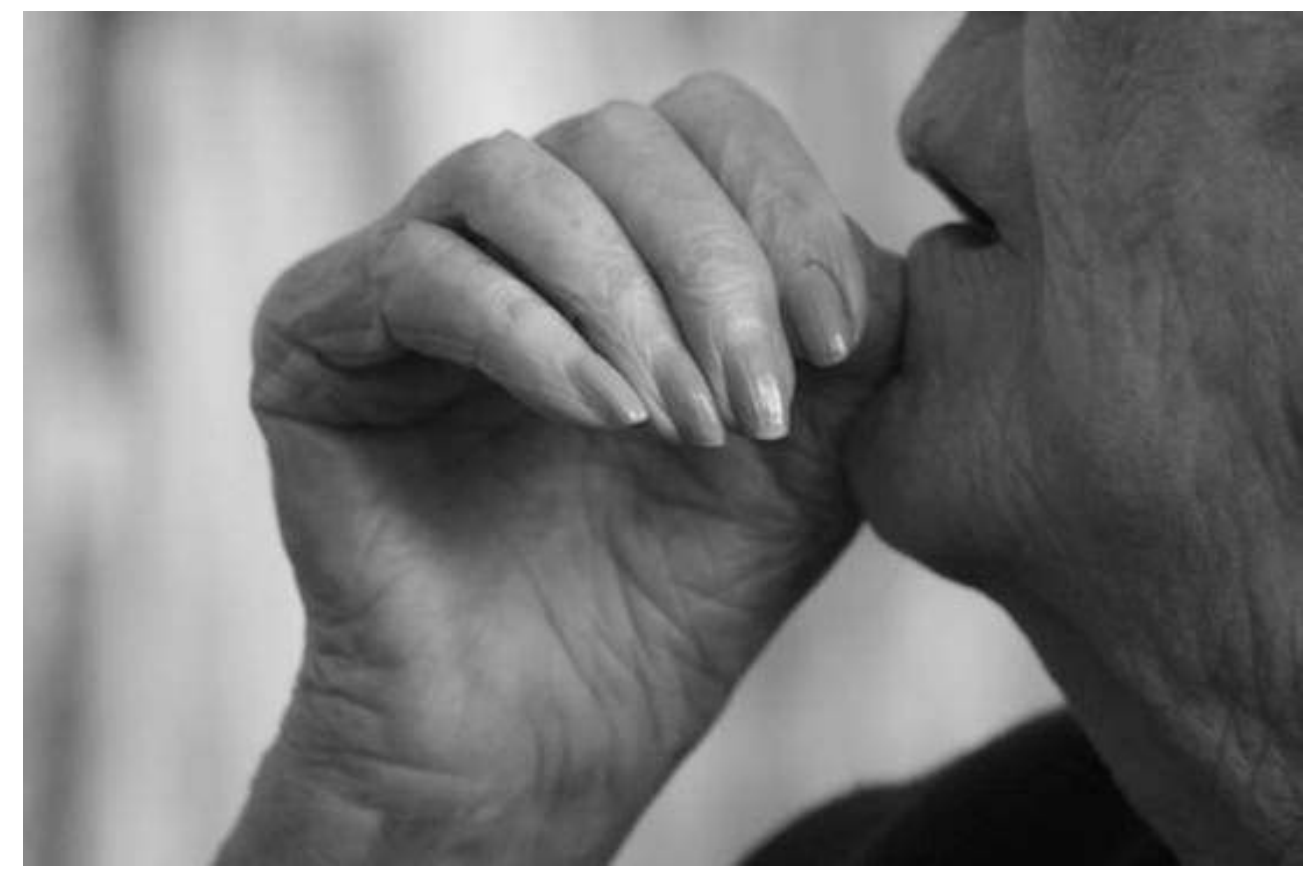

Mme $K$, dans la salle à manger, est installée dans son fauteuil roulant. La télévision est allumée mais elle semble se perdre dans ses pensées. Atteinte de la maladie d'Alzheimer, les bruits de l'environnement ne semblent pas la déranger.

11 Contrairement à Mme K, Mme J ne souffre pas de troubles liés à la maladie d'Alzheimer. Le fait d'attendre mène Mme $\mathrm{J}$ à s'attarder dans ses pensées, elle reste en lien avec son environnement mais diffère son attention. Elle s'ajuste par la distraction à la nécessité d'attendre. Par contre, Mme K semble loin de la réalité, ses désorientations l'absentent du monde et chaque moment passé dans l'attente la plonge en dehors du présent. Attendre mobilise la patience et, pour d'autres personnes, peut conduire à l'errance.

Les attitudes se conjuguent au pluriel et certaines personnes se replient entre leurs mains. Le corps devient un refuge, un repli face à un lieu dans lequel on ne peut se reconnaître, dans un environnement où les repères ne nous retiennent plus.

Mme $\mathrm{P}$ est arrivée récemment, elle pleure fréquemment et ses plaintes sont nombreuses, répétées et prolongées. Prostrée, elle restera ainsi en salle à manger jusqu'au goûter. Face à cette profonde tristesse, l'équipe se sent impuissante. 
Figure 5. Tristesse et effondrement

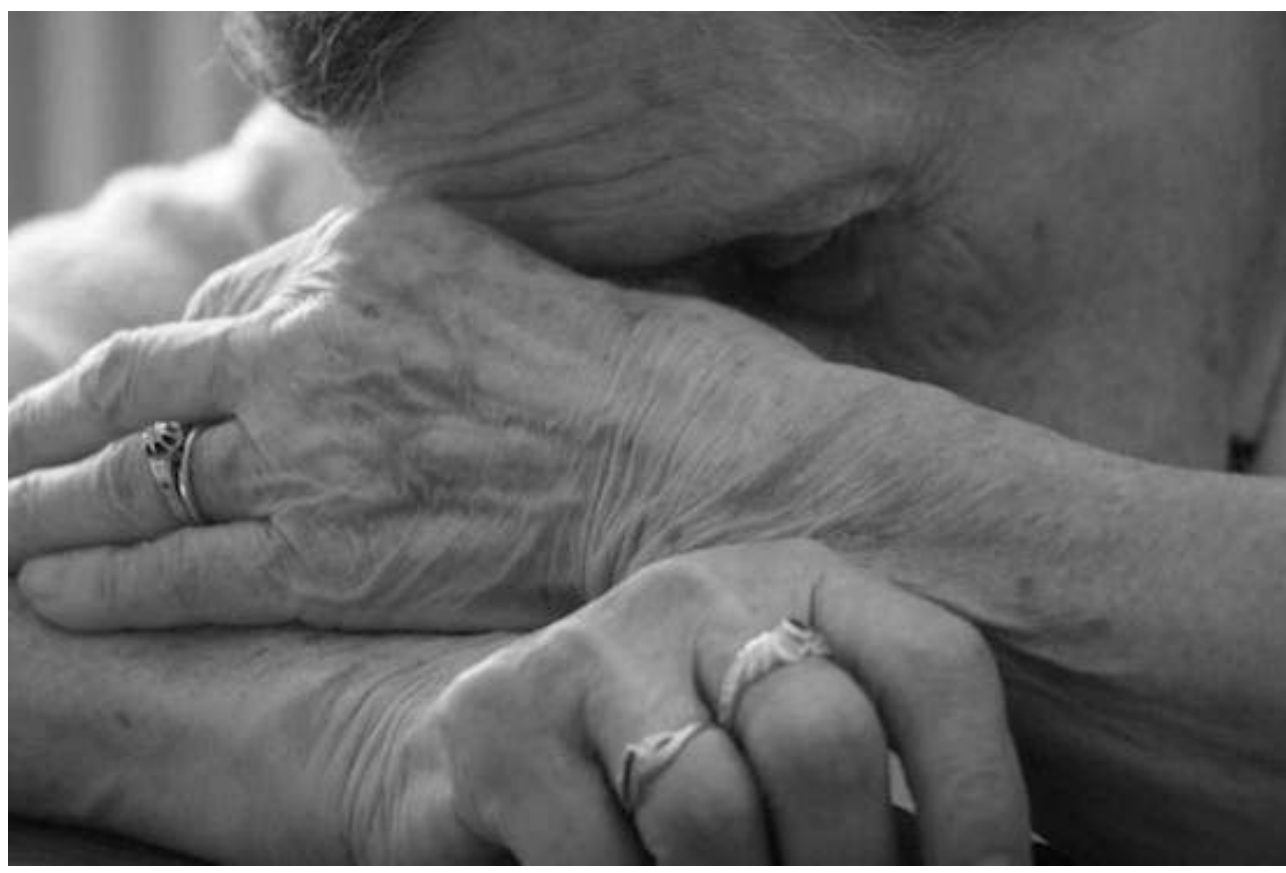

Privées lentement de l'usage ordonné des mots, les personnes atteintes de la maladie d'Alzheimer se retirent dans une mémoire émotionnelle restée intacte. Le corps, récepteur et émetteur sensoriel, se fait inlassablement le passeur de leur présence comme l'antre de leur absence.

14 Chaque photo note une attitude singulière au sein d'une situation particulière dans ce lieu de vie pour personnes âgées dépendantes. Aux formes d'attente chez les résidentes et résidents, s'y accolent celles des professionnels.

15 Lucie, aide soignante, attend les bras croisés dans la salle commune. En alerte, elle se rend ainsi disponible à toute sollicitation. 


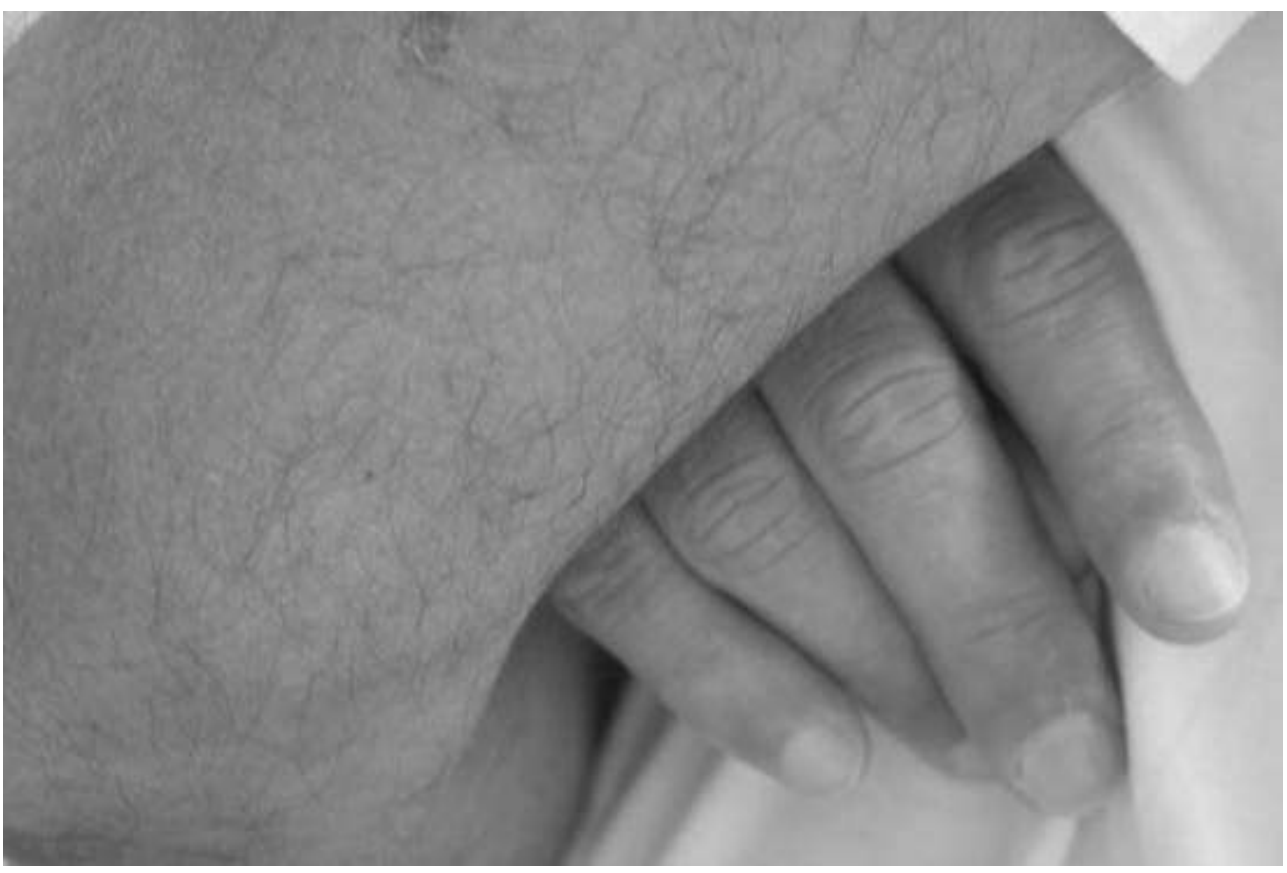

Cette posture professionnelle va de pair avec la part contingente de leur suppléance. La vie institutionnelle se compose de rencontres, d'interfaces dépendantes d'un besoin et d'une disposition d'accompagnement. Le corps soignant tend à répondre à des sollicitations d'un corps souffrant qui réclame une aide. Cependant, si cette suppléance s'instaure dans un contrat de soin à l'autre, elle est parfois redoutée.

Ainsi, à travers les gestes et les postures, la suppléance quotidienne s'anime d'actions et de réactions. Cette mise en interaction répond à un environnement particulier des troubles de la maladie d'Alzheimer que l'intention médicale tente de circonscrire.

\subsection{Des gestes adaptés}

17 Dans l'image fixe, le geste de soin apparait se décaler du geste ordinaire. Pour suppléer, la main se prête et devient pour un moment celle de l'autre. Elle se dégage alors d'une manipulation pratiquée pour soi, pour se donner à l'autre. L'acte d'aider nécessite une transformation du geste lui-même afin qu'il passe d'un corps à un autre, créant ainsi cette posture significatrice du soin.

18 La main de Gaëlle, l'infirmière, replace le bas de Mme B après un changement de pansement. Le poignet se casse pour épouser la courbure de la jambe. 
Figure 7. Pose d'un bas

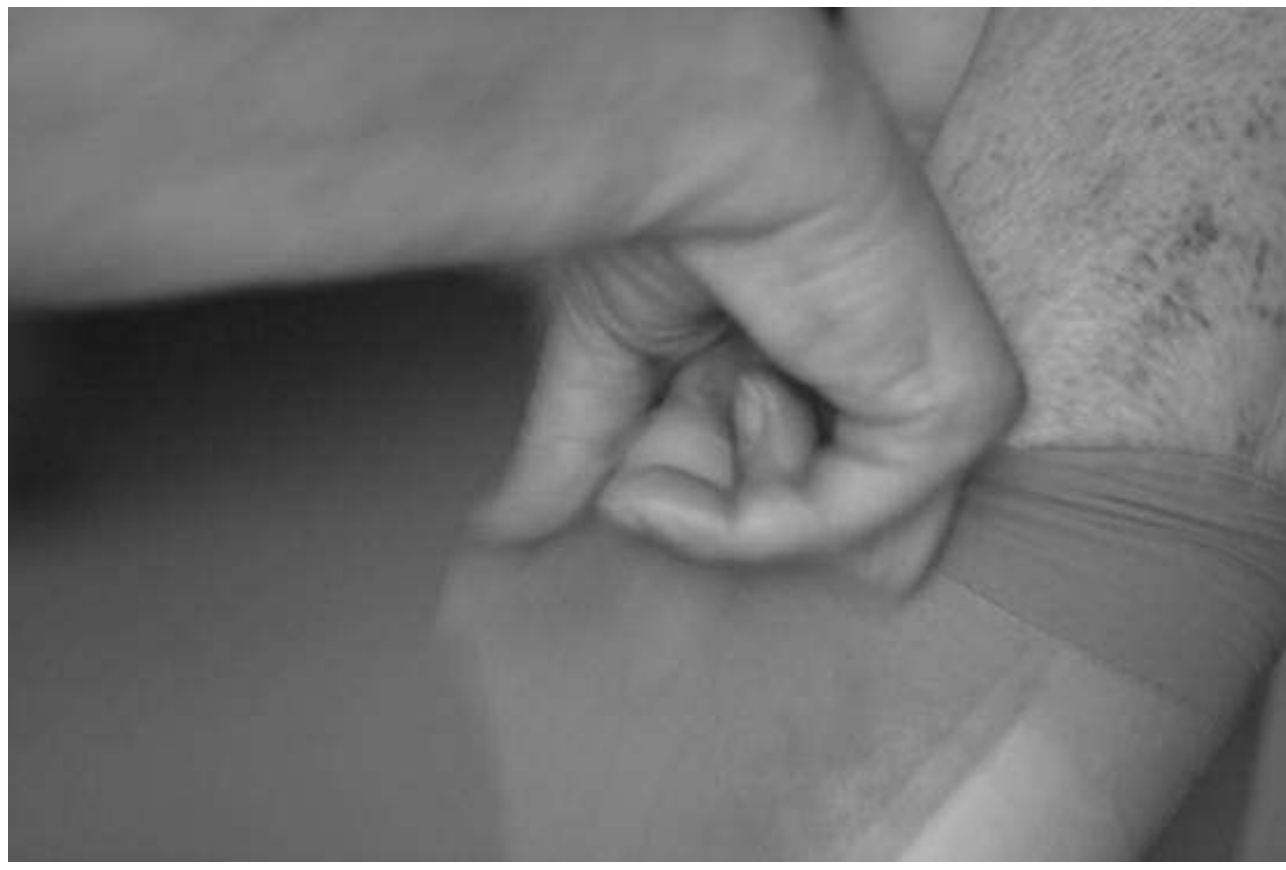

La main du professionnel se plie, se contorsionne pour s'ajuster à autrui. Cette flexibilité manuelle transforme le mouvement et renvoie à la modalisation d'un geste premier qui serait celui réalisé sur son propre corps (Goffman, 1991). Cette modification s'opère en changeant de cadre, le geste s'adapte en s'expérimentant et se perfectionne en se pratiquant. À travers les gestes, les corps se singularisent dans des postures de soignant et de soigné et tracent les contours d'une relation animée qui s'apprend. Chaque geste du professionnel semble un complément, un régulateur de plus en plus intime des failles liées à la maladie.

\section{Figure 8. Laçage de chaussures}

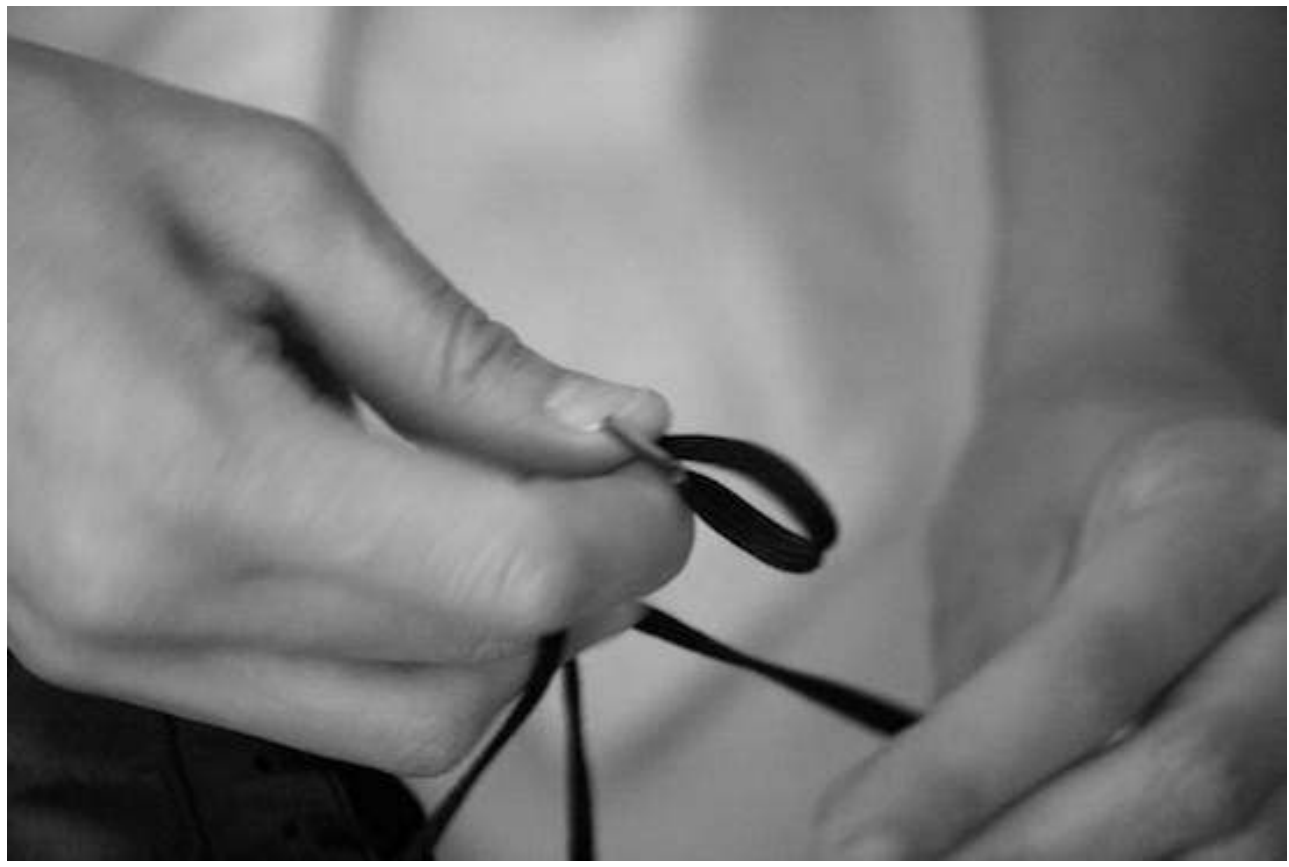

Bénédicte, aide médico-psychologique, lace la chaussure de Mme R qui ne peut plus le faire. 


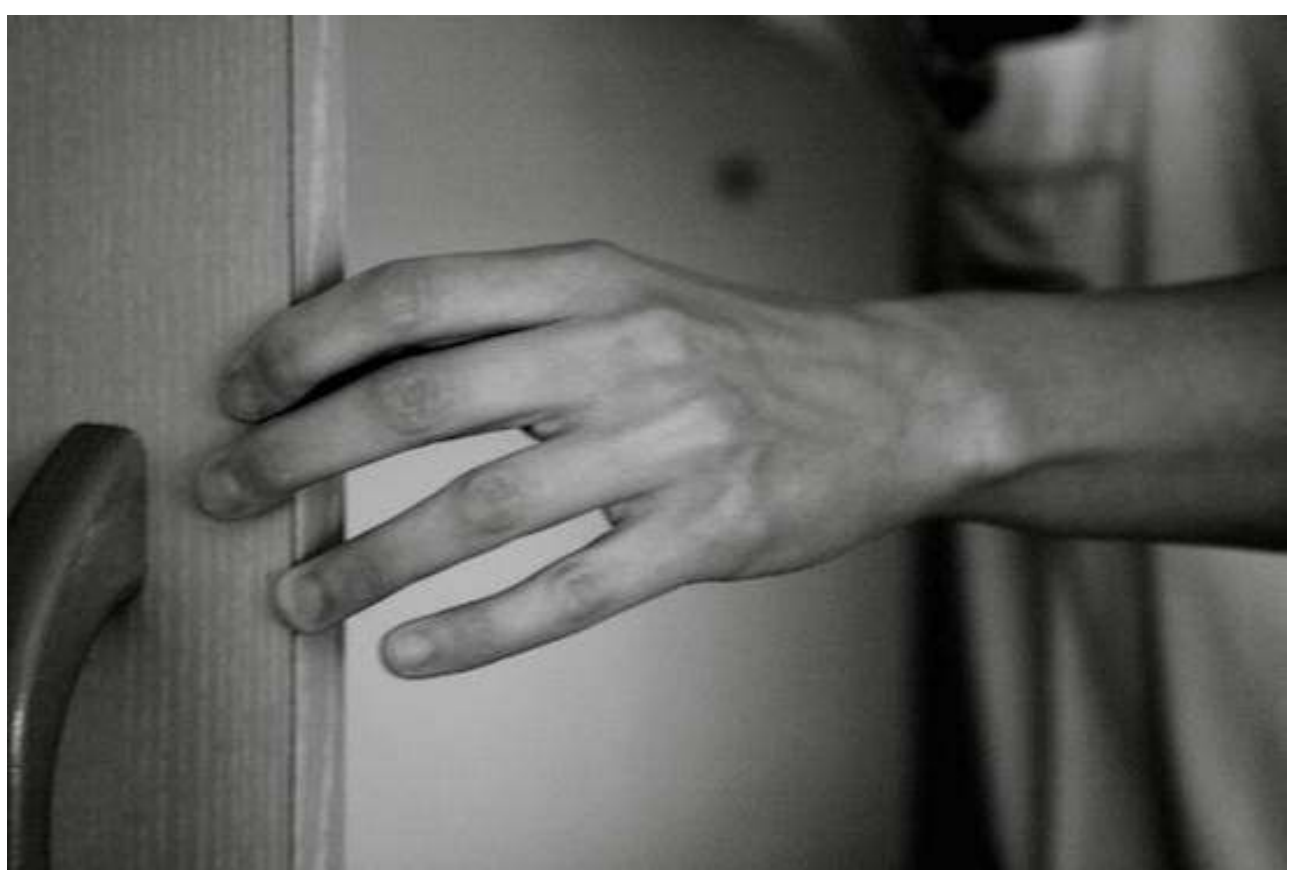

Bénédicte range les vêtements de Mme R dans l'armoire de sa chambre car, en fauteuil, il lui est impossible d'accéder aux étagères.

La toilette de Mme R, ce matin-là, aura duré 45 minutes. Bénédicte l'a accompagnée dans sa préparation corporelle jusqu'au rangement de sa chambre. Elle s'est fait le prolongement du corps de Mme R qui, dans son fauteuil roulant, la suit du regard. Par ses paroles, précédant les gestes, Bénédicte entraînait la résidence à participer à l'action par l'acquiescement à cette suppléance proposée dans son espace privé.

\section{Technique photographique et gestes de soin}

Dans les sillons d'Albert Piette qui développe une similitude entre la pratique photographique et la démarche ethnologique (Piette, 1992), la création d'images dans cette expérience a mis en correspondance une technique photographique et une pratique professionnelle. L'appareil, en tant qu'objet, a joué un rôle de médiateur entre le chercheur et son terrain. Et le procédé photographique a concrétisé cette entrevue par une mise en image. Les rapports se sont expérimentés à travers la technique utilisée.

21 Le cadrage s'est imposé comme le premier déterminant et les éléments de réglage, tels que la vitesse et la profondeur de champ, se sont pliés aux sujets photographiés et au contexte observé. Chaque situation photographiée a donné lieu à une négociation autour de choix, d'obligations et de compromis techniques en fonction des mains rencontrées. Ainsi, la création de l'image résultait d'un procédé photographique mis à l'épreuve par les gestes réalisés.

Chaque cadrage me livrait une part de cette relation particulière entre un soignant et une personne-soignée. La méthode photographique a été d'une grande importance et les diverses manipulations (souvent par tâtonnement) ont focalisé mon attention sur la variété des interactions sensibles afin d'en capter leur réceptivité. 
Cette correspondance s'articule autour de quatre points : le plan, l'axialité, la lumière et le cadre.

\subsection{Le plan}

Le format centré et resserré invite l'attention visuelle à se fixer sur les mains, une façon de s'arrêter sur ces manières de faire au quotidien. Le détail est ainsi focalisé, et livre des éléments de contact qui passent inaperçus (Piette, 1996).

La main s'outille d'objets. Ici, la « lavette » objective le geste dans un rôle particulier. La main de Claire lave le plateau de la table.

Figure 10. Main qui lave et essuie

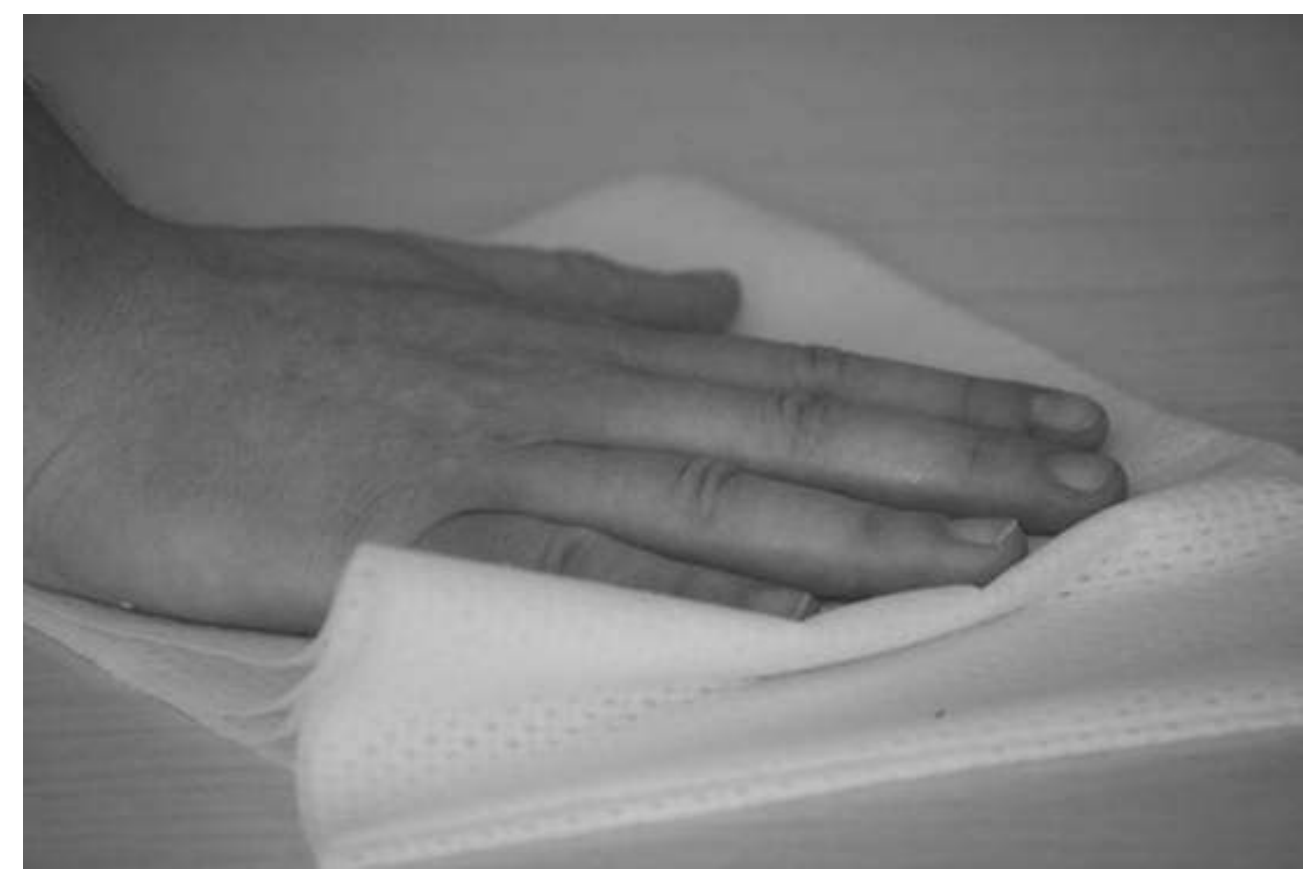

Ce geste banal de nettoyage réalisé par Claire, modifie le support-table pour le rendre abordable et plus accueillant. L'entretien du mobilier, fait par les mains outillées des soignants, concourt à l'usage du lieu. Le professionnel prévoit, anticipe, entretient ... il étaye un entourage pour le rendre familièrement utilisable. II participe ainsi à la mise en habitation de l'espace.

Comme un guide, la barre de circulation soutient Mme P dans son déplacement. 
Figure 11. Mains guidées par une barre de circulation

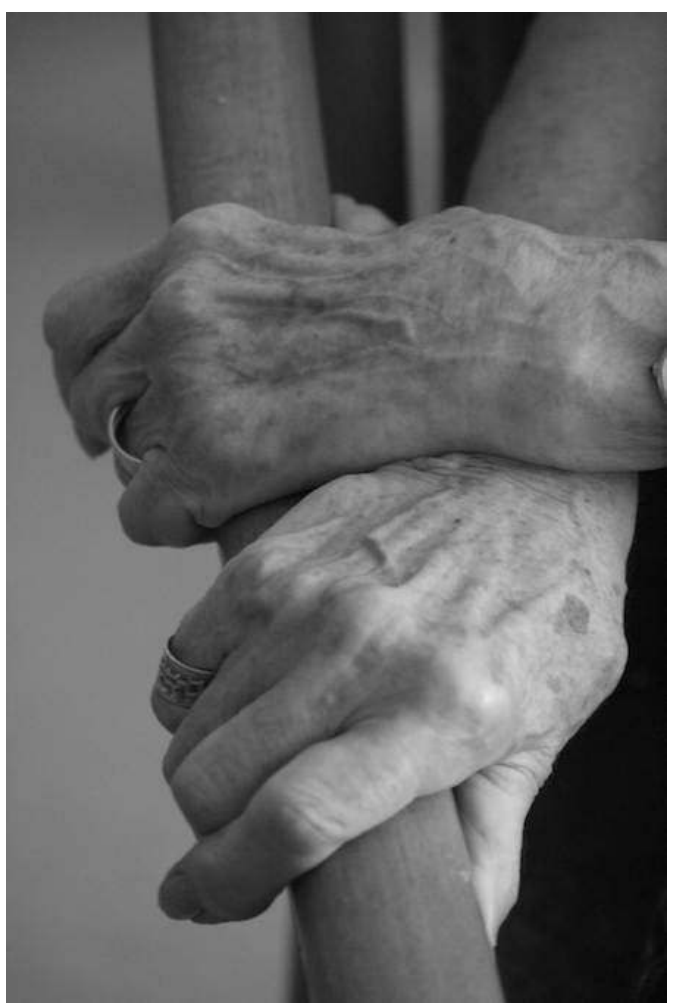

La valeur ergonomique du matériel favorise, ou non, l'autonomie et la mobilité des personnes, tout comme l'espace s'accommode par l'« entre-tient ». La qualité de l'environnement s'inclut dans un système de liaison et de soutien qui répond aux besoins des personnes dépendantes de s'y maintenir, de s'y mouvoir confortablement et assurément.

\subsection{L'axialité}

Une posture corporelle s'accorde à la prise photographique en fonction de l'image souhaitée. L'axe du regard, via l'appareil, a été principalement horizontal, ce qui a mis en évidence la présence du support.

28 Mme L sommeille dans le fauteuil devant la télévision. Sa détente corporelle s'obtient par le confort du siège que peut nous suggérer par cette main posée sur l'accoudoir. 


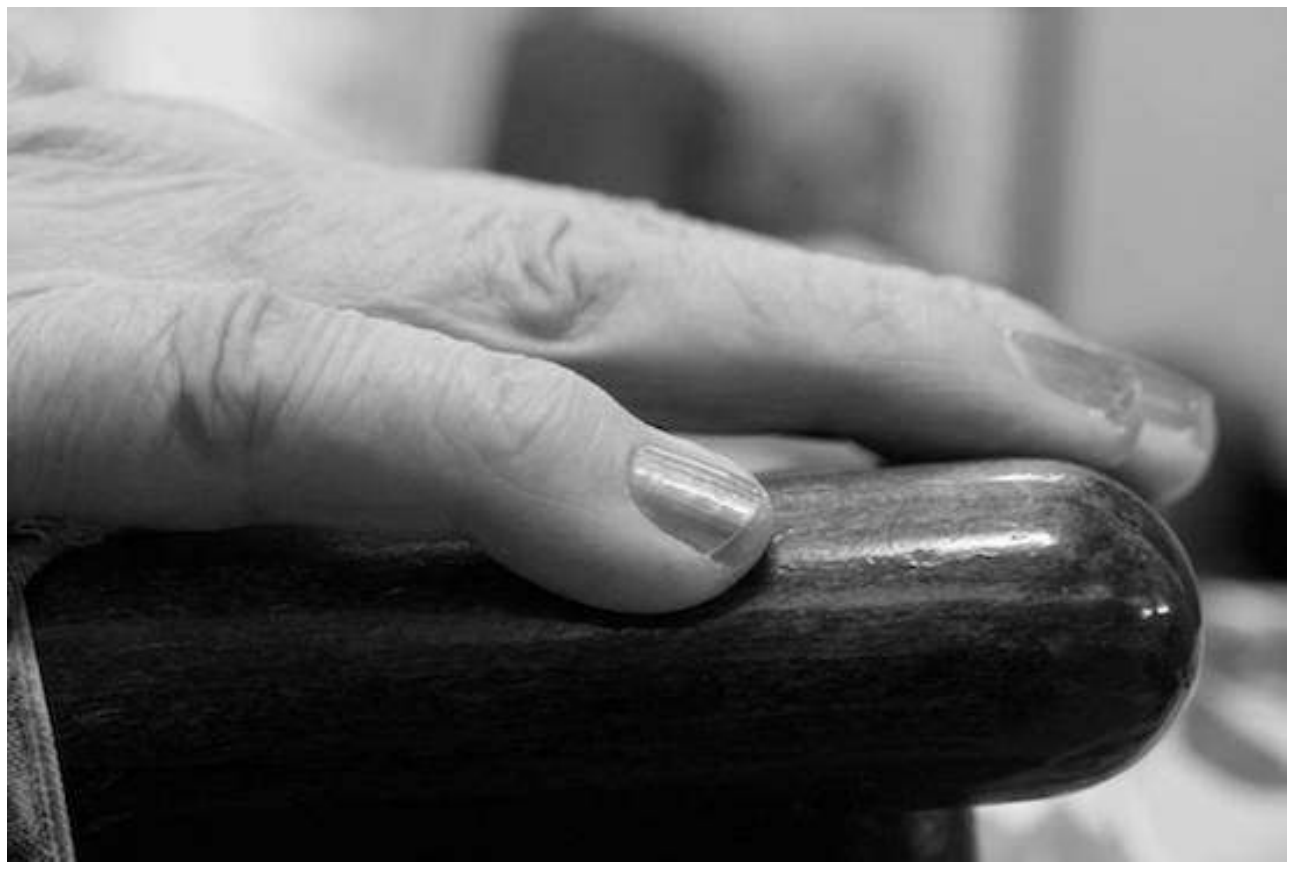

L'image fixée sur la main permet ici d'observer la posture d'aisance de la main trouvée sur le bras du fauteuil. Le confort physique s'obtient-il grâce à une interface entre surface dans une négociation de force ? La résistance, ici du support, permettrait un échange de tensions, et le confort se réalise au détriment d'un délestage de poids.

\subsection{La lumière}

Le choix d'utiliser uniquement la lumière naturelle ou ambiante pour réaliser les photographies a imposé des réglages particuliers. La lumière, indispensable à la prise photographique, a été parfois manquante dans les espaces de l'établissement. Seuls les mouvements lents trouvaient une netteté en augmentant l'ouverture du diaphragme, ce qui diminuait la profondeur de champ. La qualité de la photo était inversement proportionnelle à la rapidité du mouvement. En expérimentant la clarté, je me suis rapprochée de la synchronisation relationnelle du soin.

Patrick, aide soignant, assiste Mme K à enfiler ses chaussures avant de l'accompagner en poussant son fauteuil roulant dans la salle à manger. Le geste de Patrick est fixe, Mme K crée le mouvement. Elle était seule actrice du fait de se chausser. 
Figure 13. Aide pour se chausser

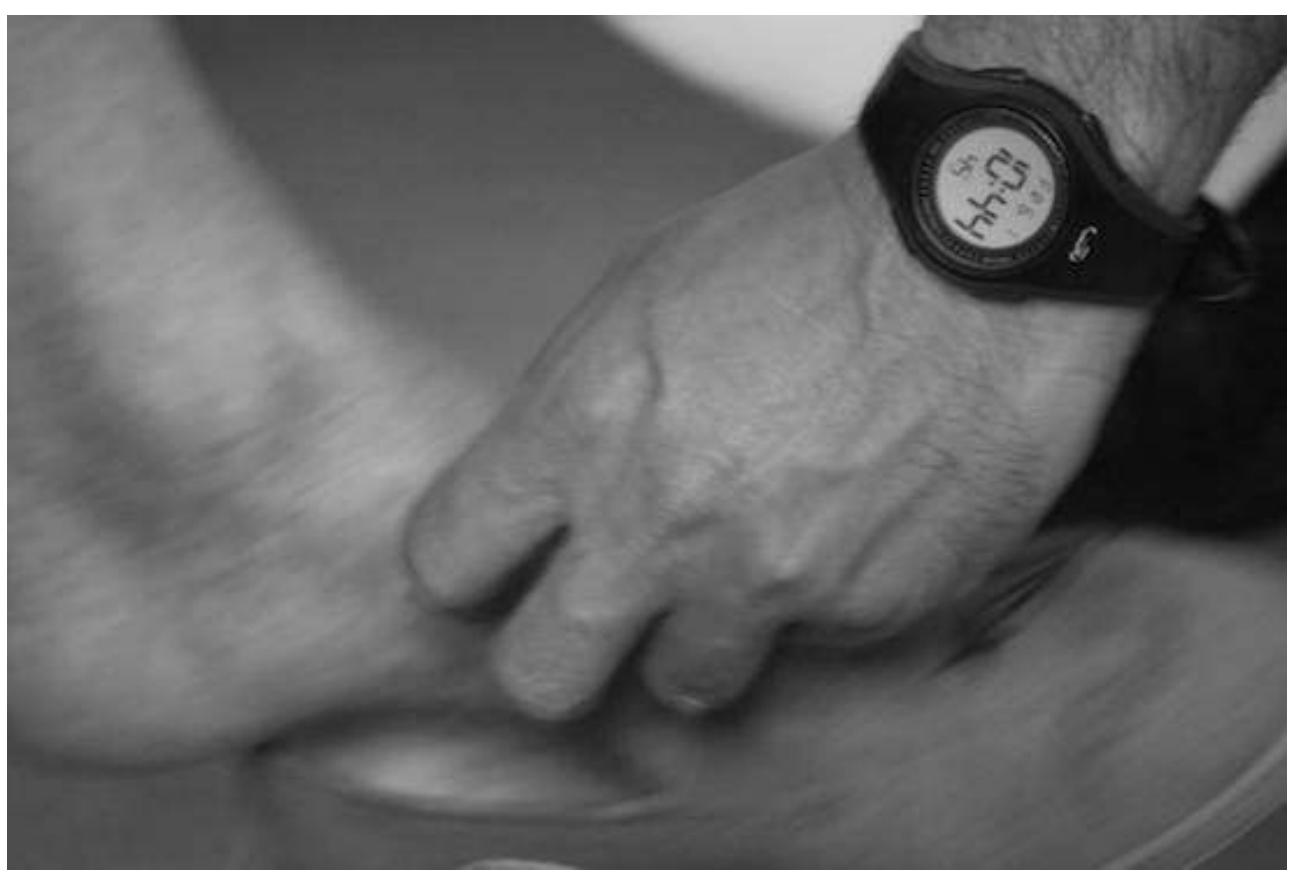

La mobilité chez le soignant et chez le soigné se complètent et s'inscrivent dans la répétition et l'alternance. Une augmentation de la mobilité de l'un semble se coordonner avec une diminution de celle de l'autre. Chaque contact soignant-soigné s'harmonise dans un rythme et un mouvement.

31 Les personnes atteintes de troubles du comportement prenaient leur repas dans un espace proche de leur chambre. Elles restaient à l'écart de la salle à manger commune où la luminosité rendait les images plus nettes. Avec l'âge, les troubles de la dextérité et les défauts de coordination sont perceptibles. La cuillère n'est pas toujours bien contrôlée et le regard des autres peut accentuer le malaise face à ce défaut d'adresse. Le fait de baisser la lumière serait-il un moyen d'effacer ses écarts de conduite ? Les zones lumineuses et les endroits sombres semblent croiser les logiques du regardable et du distinguable.

\subsection{Le cadre}

32 Le cadré centré sur les mains permet de fixer l'attention sur un geste mais, pour observer l'ensemble d'une activité, une succession de prises de vue était nécessaire afin de montrer l'agencement gestuel. 
Figure 14. Série de photos sur le passage de crème de Mme $\mathrm{P}$ par Sonia, la psychomotricienne
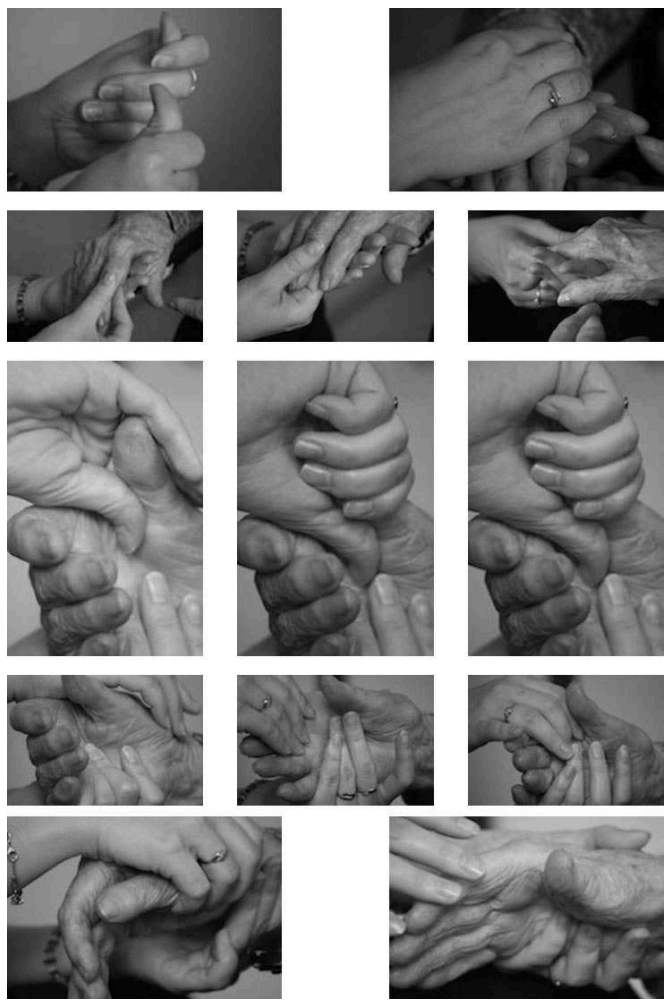

Sonia, psychomotricienne, masse les mains de Mme $P$, au stade sévère de la maladie d'Alzheimer. Ses gestes sont précis, elle module ainsi ses caresses par des stimulations adaptées, puisées dans sa formation

Puis elle recommence pour la main gauche:

Figure 14, suite
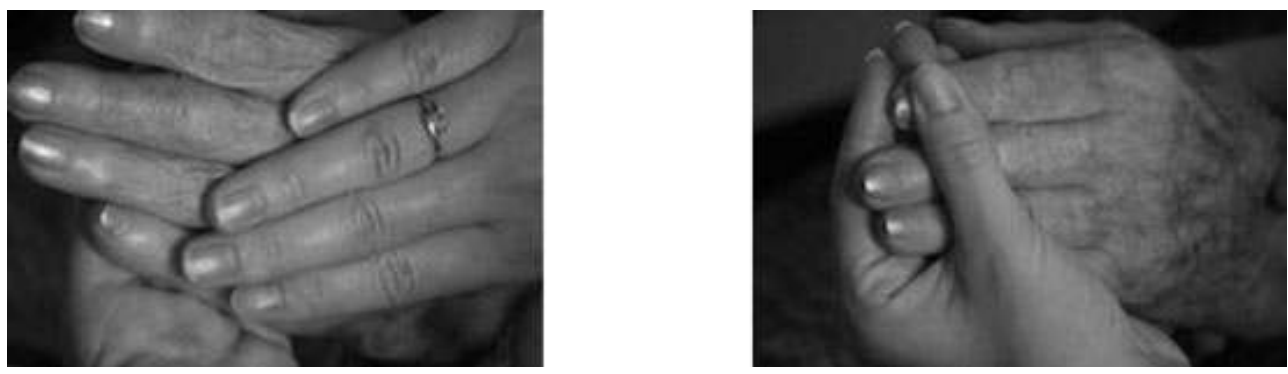

Cette série de photos met en évidence l'agencement gestuel et la progression du massage. Moins précis que pourrait l'être une vidéo, cette série n'a pas de valeur éducative mais témoigne d'une rencontre de longue durée où les mains dialoguent.

Pour Sonia, psychomotricienne, la crème adoucit la peau de Mme $P$ tout en activant ses perceptions proprioceptives mises à défaut par les contractures liées à la maladie d'Alzheimer. La stimulation tactile, par les bouts de ses doigts, sert à maintenir les facultés sensori-motrices de Mme P. Ce travail de psychomotricité entretient les voies sensibles d'une communication non verbale. L'agencement gestuel guide ce cheminement de l'un vers l'autre dans lequel la main soignante devient l'instrument de cette relation. Mais tout au long de ce soin, les doigts de Sonia conservaient le contact. Mme $\mathrm{P}$ appréciait et en retour ses mains cherchaient à caresser celles de Sonia. La 
relation s'anime d'un processus dans lequel les corps s'engagent et se côtoient mutuellement.

L'image fixe induit un cadrage qui met hors-cadre certains éléments importants pour comprendre le geste photographié.

Figure 15. Aide aux gestes du repas

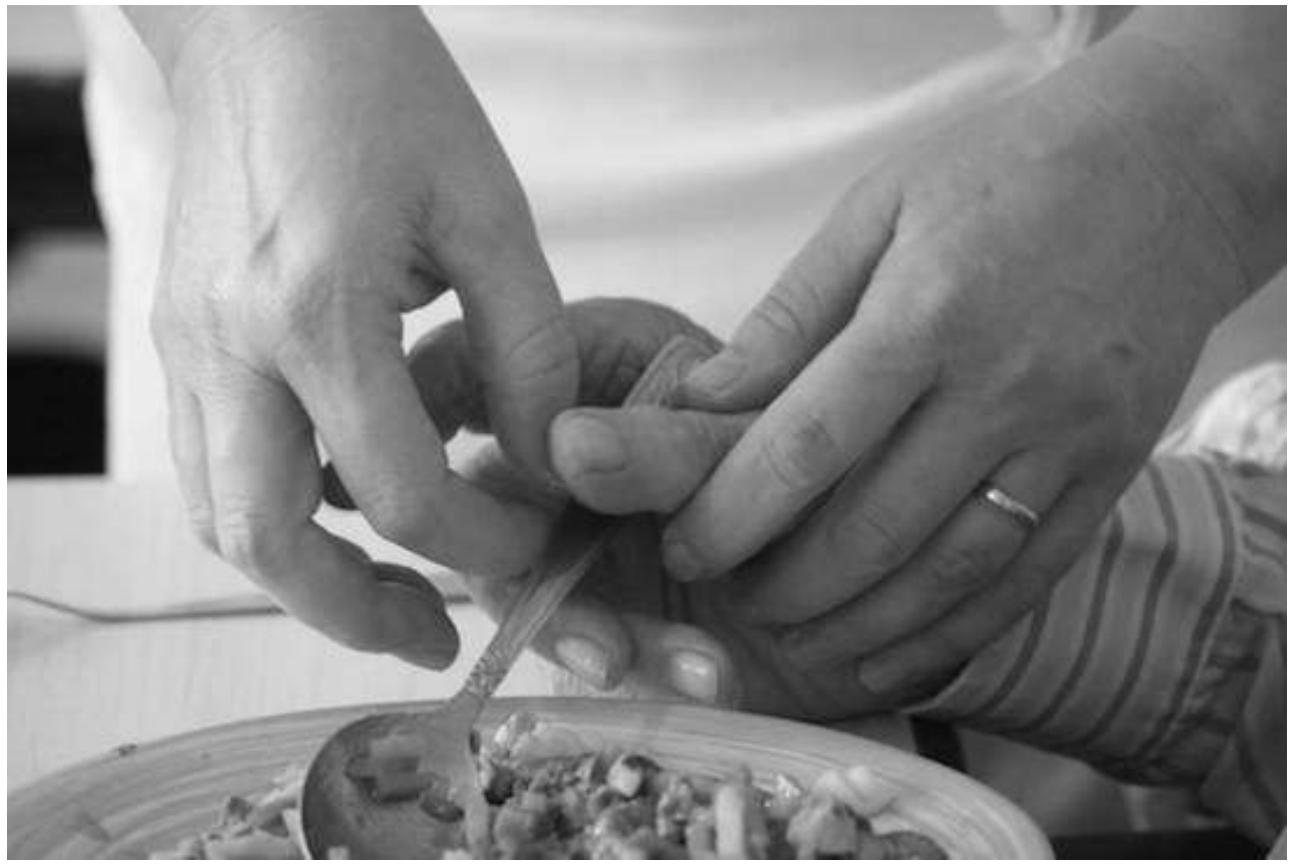

Sonia, aide-soignante, 10 ans d'ancienneté, met la cuillère dans la main de M. M pour réenclencher l'acte de manger. Placé en face d'une dame qui mange seule, M. M va pouvoir, par mimétisme, retrouver le geste.

Ici (figure 15), Sonia ne fait pas à la place, elle organise la main de M. M pour impulser la gestualité qui sera guidée par celle de la personne devant lui. Par mimétisme, l'acte de porter la cuillère installée dans sa main retrouvera la direction de sa bouche (mémoire procédurale). Par cette mise en condition, la situation du repas se crée et invite $\mathrm{M}$. $\mathrm{M}$ à manger.

Ainsi, chaque geste soignant trace un espace de potentialités pour que les personnes s'en saisissent. Les opportunités émergent d'un assemblage de circonstances, c'est sur cette création d'affordances que s'enracine l'intention soignante.

De nombreux gestes apparaissent sans signification ou d'une utilité peu notable, cependant ils semblent induire et conditionnent davantage qu'ils ne produisent en premier lieu. 
Figures 16 et 16 bis. Entretien de lunettes au moment du repas
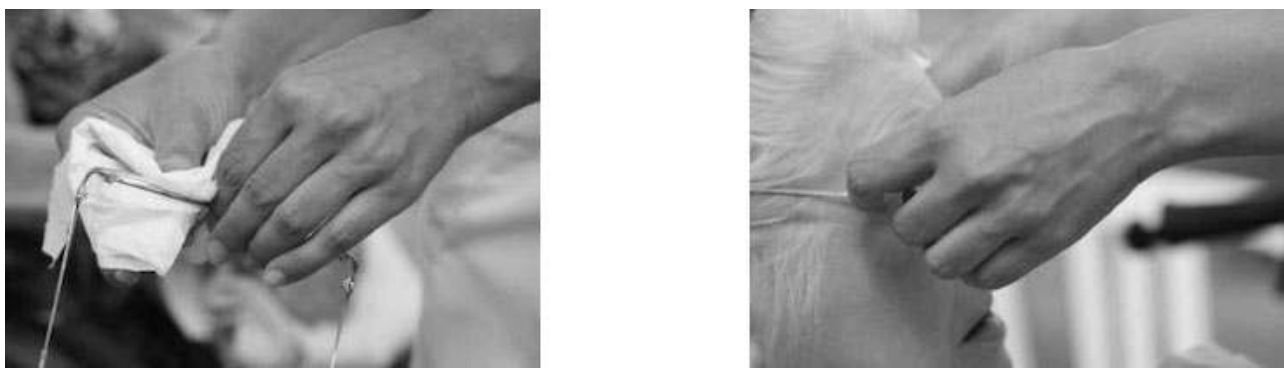

Marion, aide-soignante de l'unité protégée, nettoie les lunettes de Mme P, installée pour le repas de midi.

Par ce geste, Marion apporte du crédit à la vue de Mme $\mathrm{P}$, atteinte de troubles parkinsoniens, pour qui tout élément visuel incongru devient une source d'angoisse et peut inhiber sa mobilité. Pour Mme P, tout défaut de vision peut entrainer des obstacles insurmontables et l'empêcher d'agir. Ce simple geste s'avère être une aide considérable pour Mme P.

Un autre geste courant et banal a attiré mon attention : le soin des ongles.

Figure 17. Soin des ongles

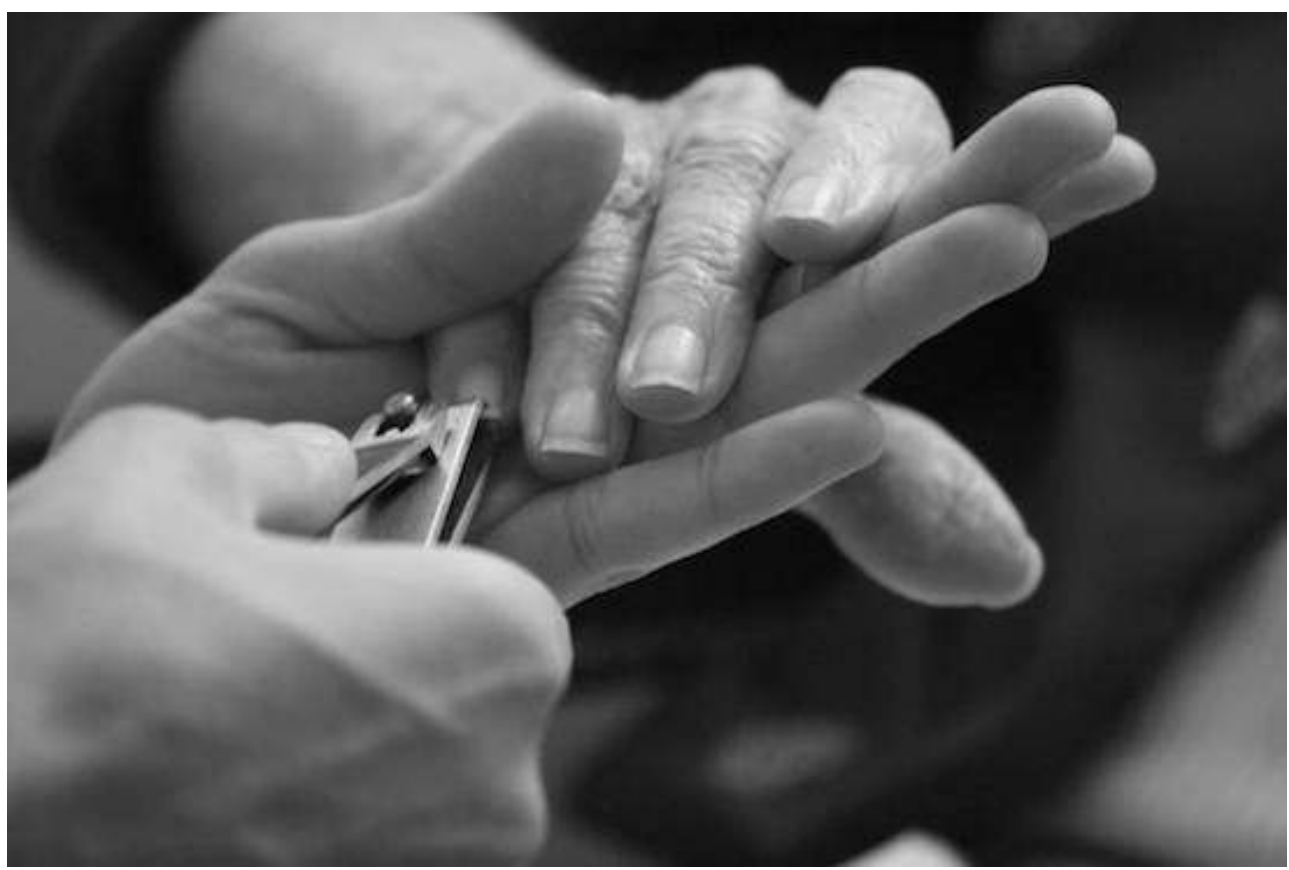

Bénédicte fait les ongles de Mme $\mathrm{R}$ durant le temps de toilette. Cette activité nécessite une approche particulière. Mme R, atteinte de la maladie d'Alzheimer, ne supporte pas de se sentir retenue. Elle peut à tout moment retirer ses mains. La formation de Bénédicte aux troubles du comportement lui permet d'intégrer ce geste commun dans un contexte lié à la maladie.

Riche de sa formation d'AMP, aide médico-psychologique, Bénédicte réalise ce geste commun en accord avec les troubles induits par la maladie. Elle limera par la suite les ongles pour en réduire les accroches susceptibles d'embarrasser la résidente. La maladie entraine des fixations de perception, et les sensations d'aspérité risquent de perturber les contacts futurs. 
43 Mais au-delà de ce soin adapté, la tenue des ongles prend une dimension plus saillante pour les familles. La tenue est un aspect important du jeu relationnel (Goffman, 1974). Ainsi, soignée, la présentation est un gage d'intérêt car elle rend le malade plus respectable et contribue à atténuer la honte qu'une négligence corporelle accentuerait.

44 Les gestes mettent ainsi en scène des jeux de placements corporels où peuvent se développer des enjeux d'influence et de manipulation.

\section{De la main « prise » en photo à la « mainmise » de l'acte soignant}

Lors d'un soin corporel, les peaux se touchent et se mêlent, la relation soignant-soigné se confond dans un portage physique et corporel où la confusion et la confrontation se jouent sur la même scène interactionnelle. La présence photographique se place en témoin de ces enjeux.

\subsection{Les manipulations}

Figure 18. Utilisation de la télécommande du lit médicalisé

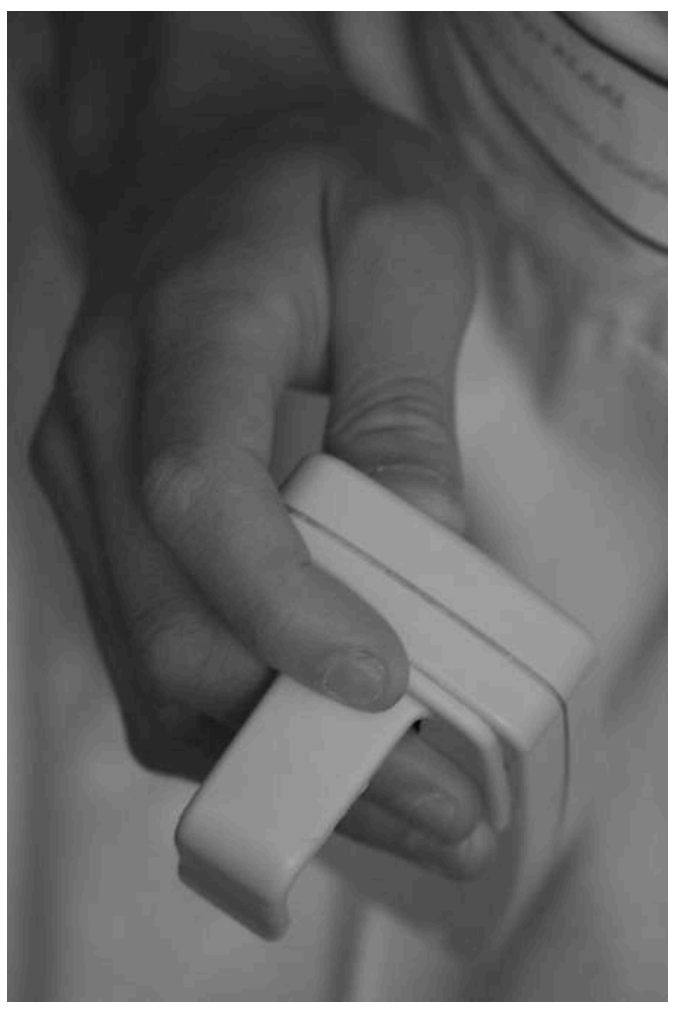

La télécommande dans la main soignante contrôle les positions corporelles des personnes dans le lit médicalisé. La maîtrise d'élever ou non un corps, de changer de position, tend à aborder l'action sous l'angle de la manipulation et de l'emprise professionnelle sur la mobilité des individus accompagnés. 
Figure 19. Distribution des médicaments

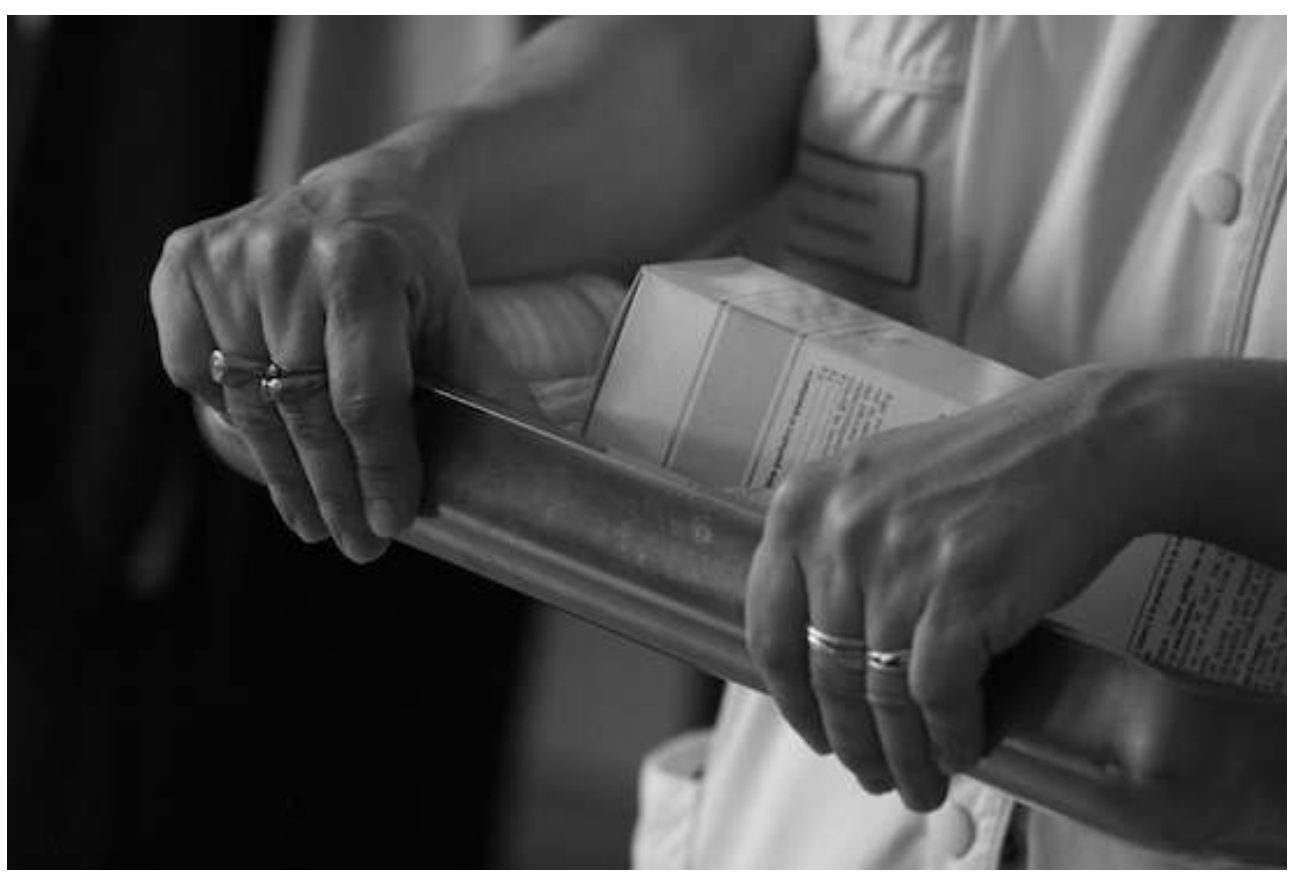

Plateau de médicaments porté par l'infirmière le midi lors de la distribution des médicaments.

46 Certains actes sont dispensés par quelques professionnels: la distribution des médicaments est réservée aux soins infirmiers par exemple. Toute une échelle professionnelle s'inscrit dans une hiérarchie des actes. La distribution d'attitudes et de postures assigne les corps à travers leurs possibilités d'agir, les contraignant à des relations déterminées.

\subsection{L'intrusion}


Figure 20. Rangement des vêtements

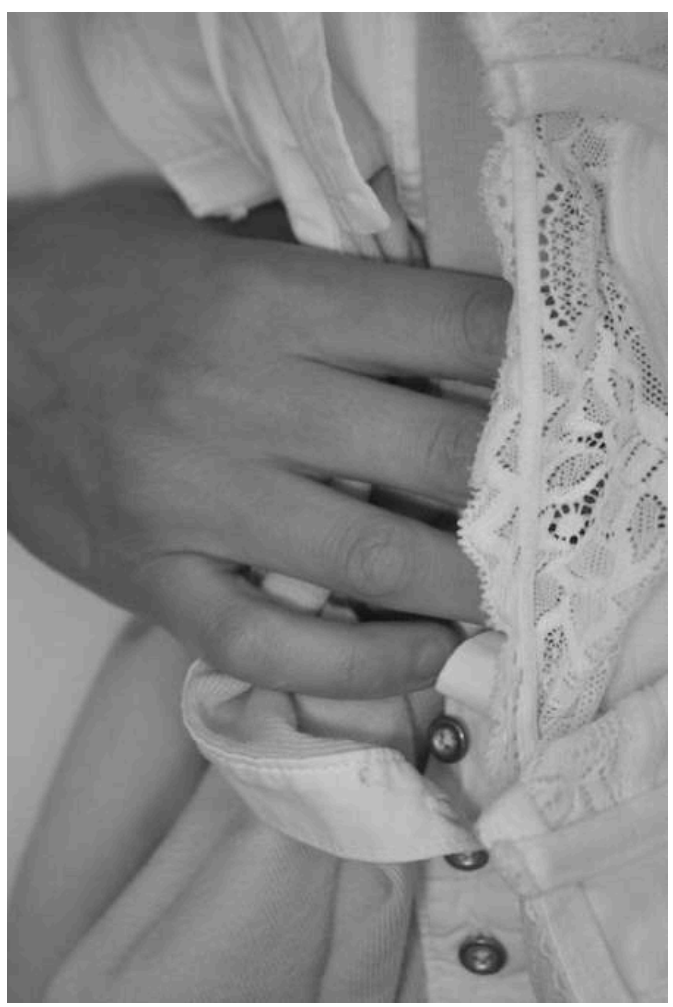

Les mains, en rangeant, s'introduisent au cœur des tissus des vêtements les plus intimes (soutiengorge).

47 La relation soignant-soigné supposerait une zone d'intimité partageable. Chaque contact serait-il le résultat d'une négociation réalisée en toute conscience ? Ou demande-t-elle un degré d'acceptation voire d'abnégation? 
Figure 21. Soin d'un dentier

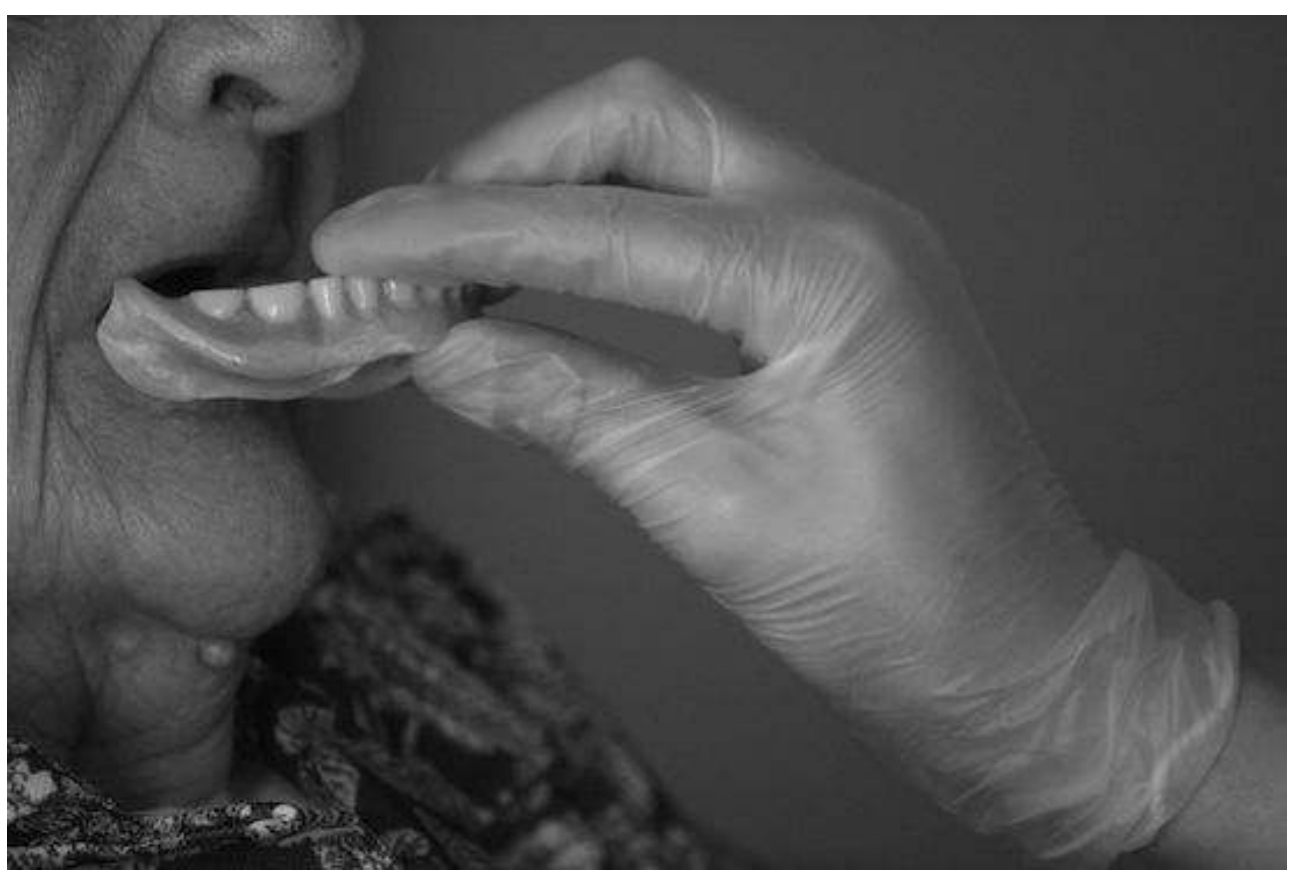

Bénédicte, AMP, place le dentier de Mme G après l'avoir nettoyé. Ce geste sera suivi d'un autre, celui de replacer correctement le col de sa robe. Puis Bénédicte accompagnera Mme $\mathrm{G}$ vers la salle à manger. Mme G, en fauteuil roulant, est apathique et mange un repas mixé.

Une part intrusive de l'acte apparaît violer les frontières de l'intime. Ces situations gênantes sont suivies de menus gestes anodins chargés d'affectivité comme l'ajustement du col de la robe de Mme $\mathrm{G}$ par Bénédicte. Les réactions viennent compenser et rééquilibrer les tensions vécues dans l'incongruité de l'acte. En injectant de la quotidienneté, ces gestes se trouvent replongés dans le temps commun qui n'effraie plus. Certains soignants espèrent presque ainsi dissiper ce ressenti de violation, en l'intégrant dans les oublis de la routine.

49 L'insupportable serait d'oublier la raison ou de réduire le sens du geste. Pour une personne non-soignante la figure 21 (ci-dessus) peut conduire l'intrusion jusqu'à l'intolérable. Ici, seule la main dans l'axe de la bouche suppose une attention symétrique et offre une ouverture sur une interprétation différente de la partie intime du soin.

\subsection{Le plaisir}

50 C'est derrière l'appareil que j'ai réalisé l'importance de cette énergie circulante de peau à peau qui procure de l'apaisement et du bien-être. 


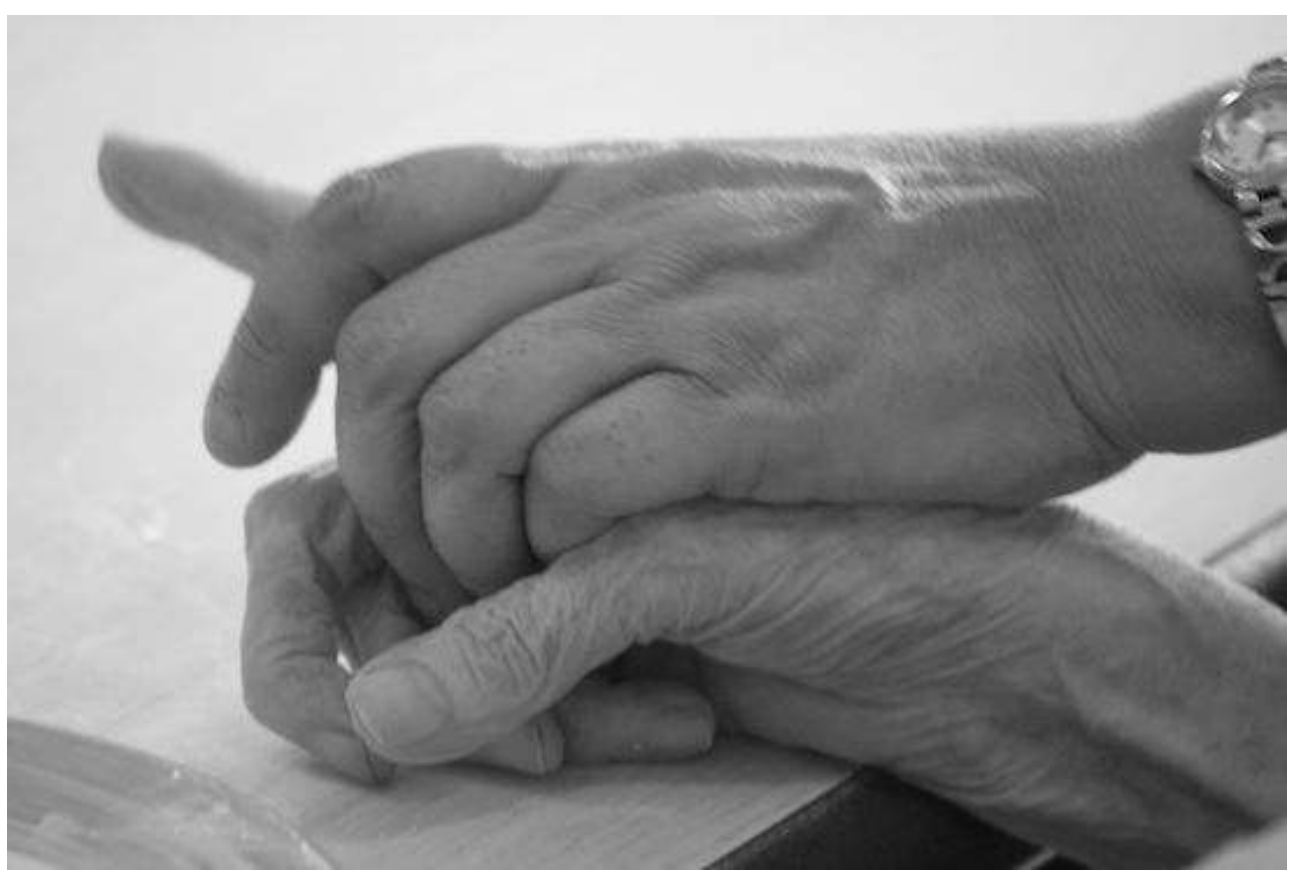

Claire, AS, prend la main de Mme G qui au moment du repas s'agite. Elle place sa main pour apaiser son inquiétude. Calme et sereine, elle prend plaisir à le faire. Elle sent que son acte est favorable à Mme G. Elle en retire une grande satisfaction.

51 La photographie ci-dessus vient confirmer ce que j'avais relevé lors des entretiens et des observations : à savoir que la satisfaction d'un contact appelle un autre contact. Le plaisant convoque le plaisant. Les gestes de tendresse et d'attention contribuent à légitimer une proximité corporelle par un attachement des personnes dans une histoire commune, une relation dans laquelle des gestes de grande intimité peuvent se réaliser. Ce procédé d'affection vient compenser l'incommodité et le malaise que fait vivre la situation de soin.

\section{Un nouveau cadrage dans d'enquête}

Cependant, la pratique photographique est-elle pertinente dans le domaine du soin? Rendre visible une relation intime ne serait-il pas de l'impudeur? L'intime est par définition privé et se déroule sans témoin, alors que la production d'image fait sortir de l'ombre ce qui se vit dans le privé. Montrer ce qui ne devrait pas l'être ne transgresserait-il pas une invisibilité structurelle (Perez, 2000) au risque d'en affaiblir son aspect fondateur?

53 Cette expérience photographique a suscité des malentendus et des rejets de la part de l'institution. La condition sine qua non du projet-photo portait sur la faculté de rendre anonymes les images, les mains semblaient y répondre mais ne paraissaient pas suffisantes. La crainte dépassait le respect de la vie privée des personnes et rejoignait celui du jugement du soin gérontologique en général et sur leur manière de faire en particulier. Les interprétations possibles face aux images préoccupaient la direction. 


\subsection{Une nouvelle négociation} d'une affichette à l'intention des équipes de soin, les invitant à collaborer au «projet- 
photos». La démarche s'intégrait comme une activité de la vie de l'institution, difficilement contestable par les professionnels. Il n'y a eu ni négociation ni refus, et l'appareil n'a pas rencontré un enthousiasme global. Sans opposition verbalisée, les désaccords de certains membres du personnel se sont exprimés de façons contournées. Certains soignants se positionnaient de manière à ne pas laisser leurs mains à la vue de mon objectif ou leurs gestes se faisaient plus rapides. Les réflexions m'ont été faites « laisse donc ton appareil et viens nous aider ! », ou encore « cela ne rime à rien! ». Sur le ton de la moquerie, ces remarques parfois piquantes exprimaient leurs propres embarras et incompréhensions face à cette démarche.

63 Sans le tissage relationnel réalisé au préalable par l'observation participante auprès des agents (ménage, toilettes, repas, etc.) et par les entretiens individuels, les réactions auraient été surement plus vives. Ce temps passé auprès des professionnels a permis d'amoindrir cette relation imposée par une information descendante. Car cette nouvelle alliance m'éloignait de leur proximité et apportait à ce regard photographique le jugement et l'évaluation.

Après un début inconfortable, et au fur et à mesure de ma présence auprès des équipes avec l'appareil, les relations se sont recréées. J'avais opté pour un appareil à écran numérique pour visualiser les images. Ainsi, dès que je le pouvais, je montrais directement les photos réalisées et relevais les réactions.

Figure 23. Main sur la nuque de Mme T

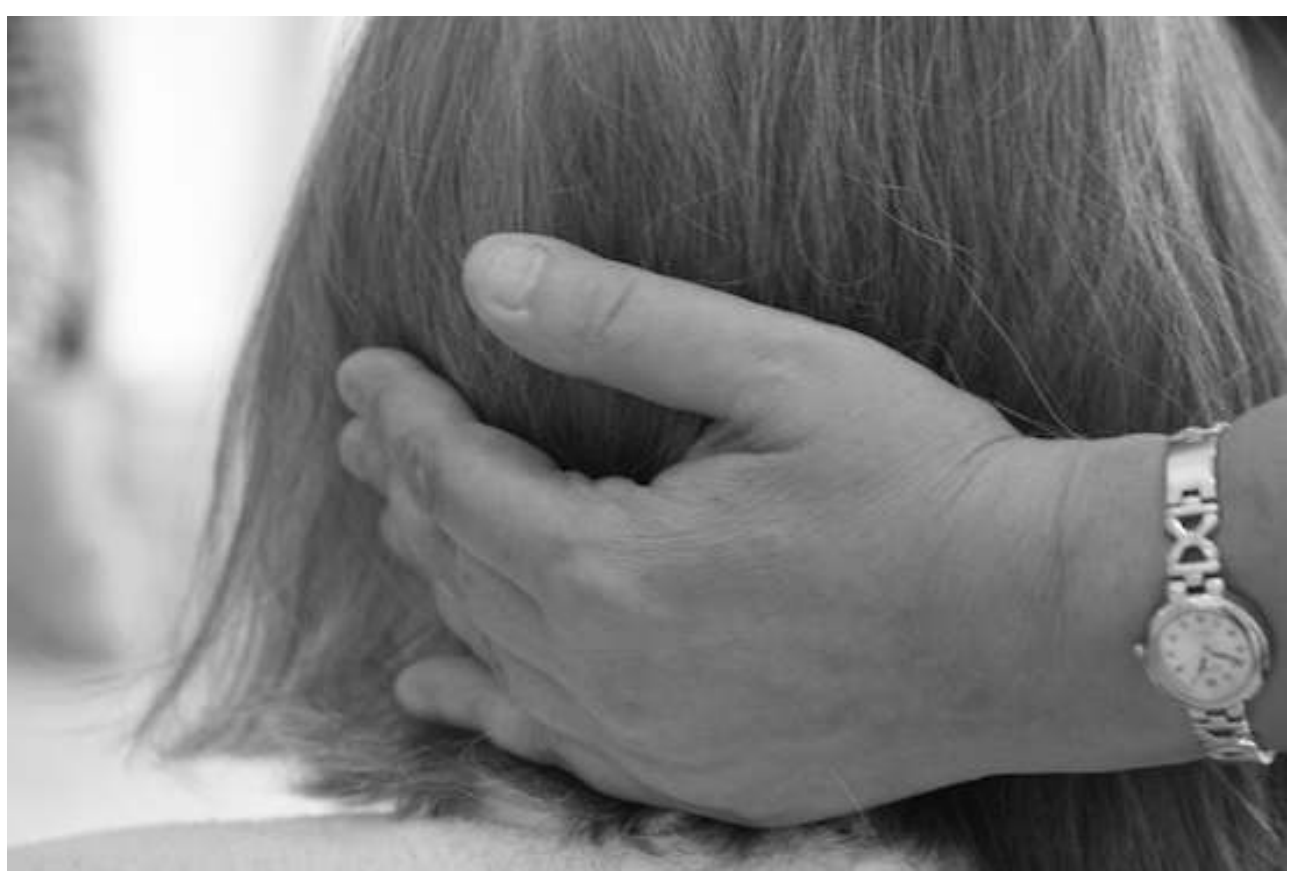

«C'est fou, c'est exactement ça ! Ma main est juste là pour donner une impulsion, une présence afın de stimuler Mme T à manger ! » (Claire, ASH - Agent de service hospitalier - en cours de VAE (validation des acquis par l'expérience) pour être AS (aide soignante), 15 ans d'ancienneté dans l'institution) - Photo prise lors d'un repas. Paroles émises à la vue de la photo.

Ici, la verbalisation au regard de la photo a permis à Claire de se voir agir. À la vue de l'image réalisée, elle a pris conscience que sa main ainsi posée faisait l'objet d'un intérêt. La photo a réifié un geste banal du quotidien en un acte professionnel qui 
devenait remarquable. À travers la mise en image, l'acte passait d'une réalisation insaisissable à une réalité intelligible.

L'incongruité de l'appareil photo a favorisé de nombreux échanges avec les personnes habitant la résidence. De la même manière, ma présence continue dans les lieux communs, nécessaire à la prise photographique, a fait l'objet de demandes diverses : aide aux déplacements, services de verres d'eau, aide pour se vêtir ou se dévêtir... . Si cette situation hors du commun et inhabituelle a suscité de la curiosité et des interpellations, rapidement ces sollicitations se sont avérées difficiles à gérer pour la pratique photographique elle-même. L'œil indispensable derrière l'objectif rendait toute conversation laborieuse.

67 Les nécessités techniques et relationnelles semblaient se confronter mais également s'alimenter pour aller plus loin dans la recherche. Ainsi, à mesure des prises de vue, s'offrait une série de gestes professionnels en images. Mon enquête ethnographique s'est prolongée en notes photographiques et en discussion autour de l'écran numérique.

Cet événement photographique a suscité des réminiscences et a fait l'objet de confidences.

Figure 24. Doigts tordus de Mme B

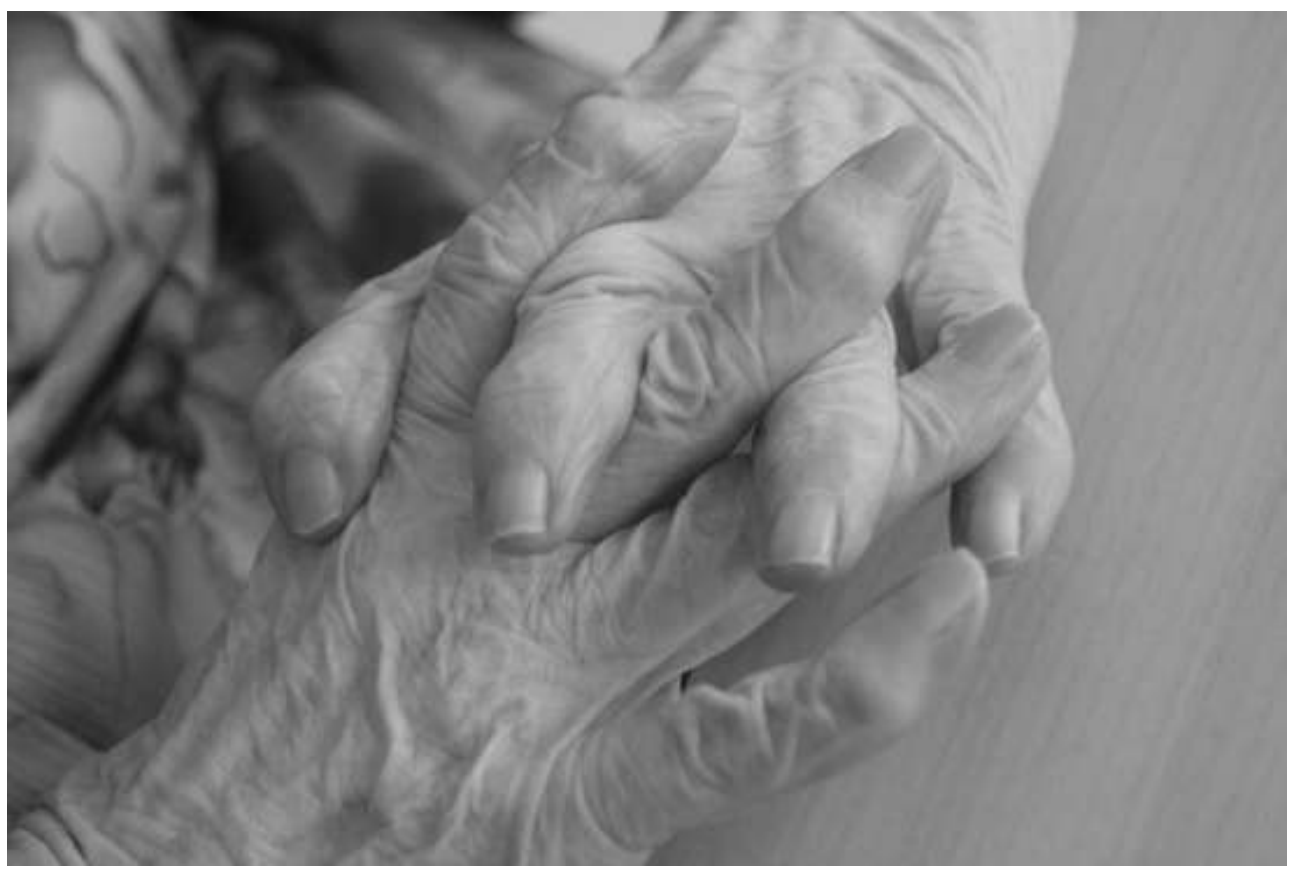

"Mes mains sont toutes tordues. Elles sont vieilles », constate Mme B avec un certain abattement. Mme $B$, résidente de 81 ans, marche avec une canne. Réaction recueillie à la vue de la photo. Mme B a ensuite engagé la discussion sur les photos de son mariage et de sa vie de famille. Me signalait-elle que ses mains avaient été également jeunes? Et belles ? Et qu'aujourd'hui elles avaient vécu?

69 L'image d'entremêlement de doigts, de stries réticulaires et des rides accentuées par le croisement des mains, a conduit Mme B à évoquer les épreuves de sa vie. Comme une trace déroulant un parcours, cette photo semble ouvrir sur la vie entière de Mme $B$.

70 La création photographique, en se fixant sur les mains, invite tout un corps individuel à se raconter. Une seule personne, résidente depuis un an, a clairement exprimé qu'elle ne voulait pas être photographiée. À plusieurs reprises elle a énoncé: «cela ne 
m'intéresse pas». Elle refusait toute implication institutionnelle et participait rarement aux activités collectives. Elle réglait ses "petites affaires » disait-elle, sans personne, sans photo, mais également sans discussion car rapidement, elle coupait court à toute tentative d'interaction.

71 Se laisser photographier serait-il se livrer? Se prêter à l'image semble exiger une certaine confiance pour se confier à l'objectif.

\subsection{Les limites}

72 Le corps est un sujet délicat et sensible. Le corps à corps de la relation de soin le devient doublement par sa condition privative. L'intimité du geste serait-elle accessible au regard étranger? La photographie construit à la fois la force du cadrage de l'image mais également sa faiblesse par ses limites contextuelles.

Le noir et blanc a été un choix, celui d'accentuer le grain de la peau. Cette transformation monochrome cherchait à trancher avec les couleurs de la vie commune, avec la volonté de placer le geste dans une distinction et l'acte dans une compréhension. Couplée au plan serré, des choix techniques ont été nécessaires pour guider le regard sur ce moment d'accompagnement. Les éléments technométhodologiques garantissent le processus de distinction de ce phénomène de proximité, afin que le déroulement de l'action se perçoive dans sa dimension unique, manuelle et charnelle.

74 Le cadrage et le noir et blanc influencent la réception de l'image. Ce nuancier de gris s'associe à une transformation esthétique du réel pour le rendre perceptible et communicable. Et les images produites préparent les gestes professionnels des soignants au regard extérieur. Car chaque élément dans la photo devient un stimulateur de subjectivité qui active la frontière avec sa propre intimité. La dimension esthétique mitige le sentiment d'intrusion et de voyeurisme face à l'intime exposé. De plus, cette transformation permet au regardant d'accommoder sa vision en travaillant ses propres perceptions. Ainsi, le voile esthétique diminue l'appréhension d'une confrontation avec un milieu méconnu ou repoussant pour favoriser l'interface et la reconnaissance.

75 Cependant, cette dimension est critiquable et met en évidence la part modélisatrice, voire manipulatrice de la technique sur les impressions sensibles. L'esthétisation conduit sur la voie d'une création de type "photo-auteur» au détriment des informateurs-acteurs. Cette confusion des intentions occulterait une mise en parole du sujet de la photographie pour fixer l'objet photographié. La présentation des images produites aux agents de terrain serait un moyen d'éviter le biais du cliché et de poursuivre la démarche ethno-photographique du proche. La valeur esthétique devient alors une donnée supplémentaire à analyser dans le cadre d'un système de protection et de discrétion et d'un accompagnement à l'expression.

76 Sous la forme d'élément plus au moins allusif, le geste dévoile des réseaux de logique qui entrainent un travail de résonances et d'interprétations. Certaines situations de " prise en soin » peuvent susciter de fortes réactions qui effleurent l'incompréhension ou la crispation chez les personnes non avisées.

77 Le métier de soignant s'inscrit dans l'intimité physique et physiologique des personnes. L'hygiène corporelle fait partie de cette relation où le convenable est dicté par l'ordre 
du tabou. Les formes allusives dans la création d'images amènent à apporter certains faits de manière figurée. Ainsi, la dimension répulsive d'une situation douloureuse ou intime se trouve transformée par un procédé métaphorique. Seule la contextualisation guiderait l'interprétation en donnant des éléments situationnels de l'acte tout en limitant la dimension fantasmatique et fabulatrice qu'éveillerait une curiosité impudique.

Figure 25. Gants de soin

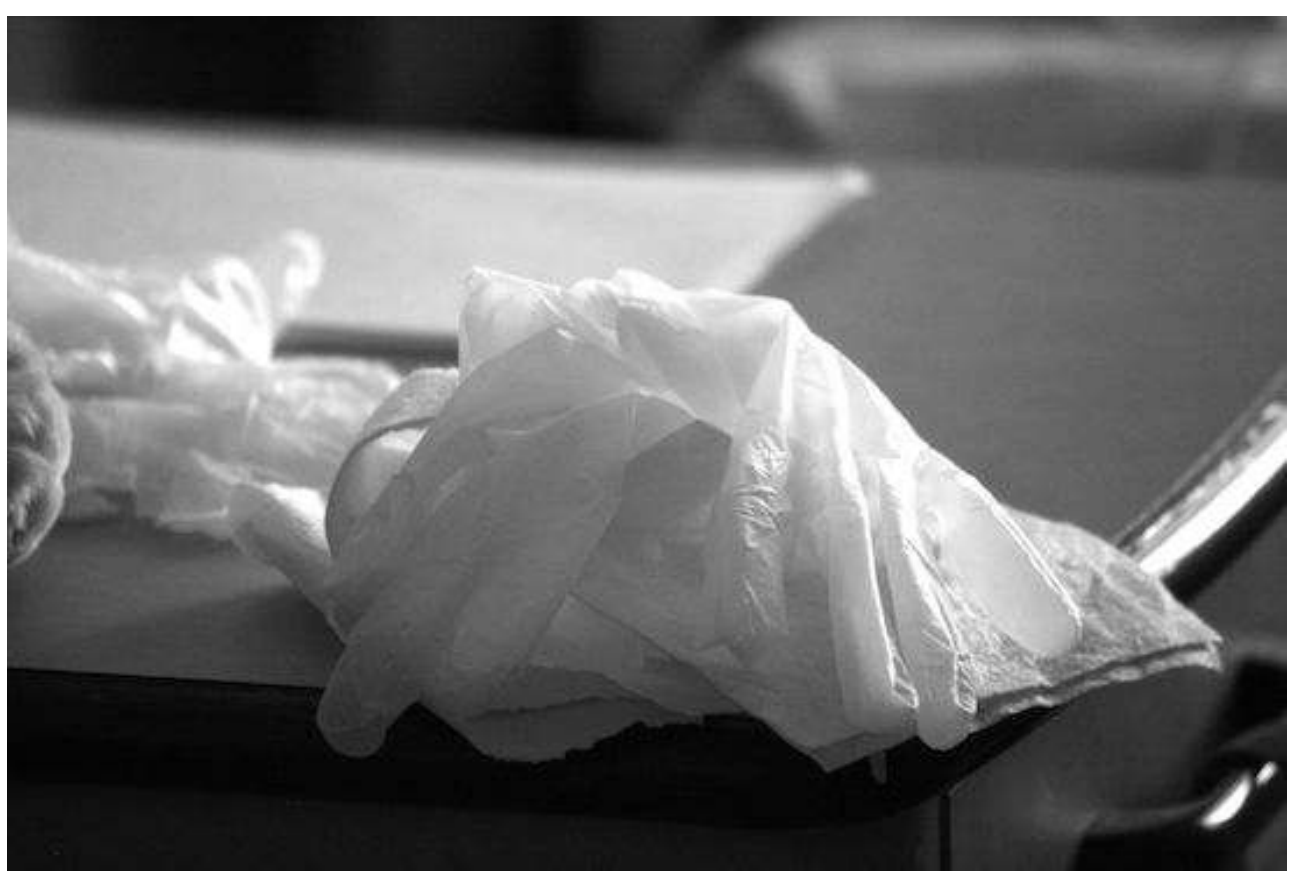

Gants enlevés en fin de toilette et laissés sur une table mobile dans une chambre d'une personne. Ils seront jetés dans la poubelle de la salle de bain au moment du rangement de la chambre par le même professionnel après avoir accompagné la personne au salon.

Ici, la figure 25 sous-entend l'acte à travers les gants. Le geste n'est plus photographié, il est suggéré. Dans l'accompagnement, les traces d'un désordre sont rapidement effacées. Le phénomène redevient invisible après que la situation ait été contenue. 
Figure 26. Confection d'un lit

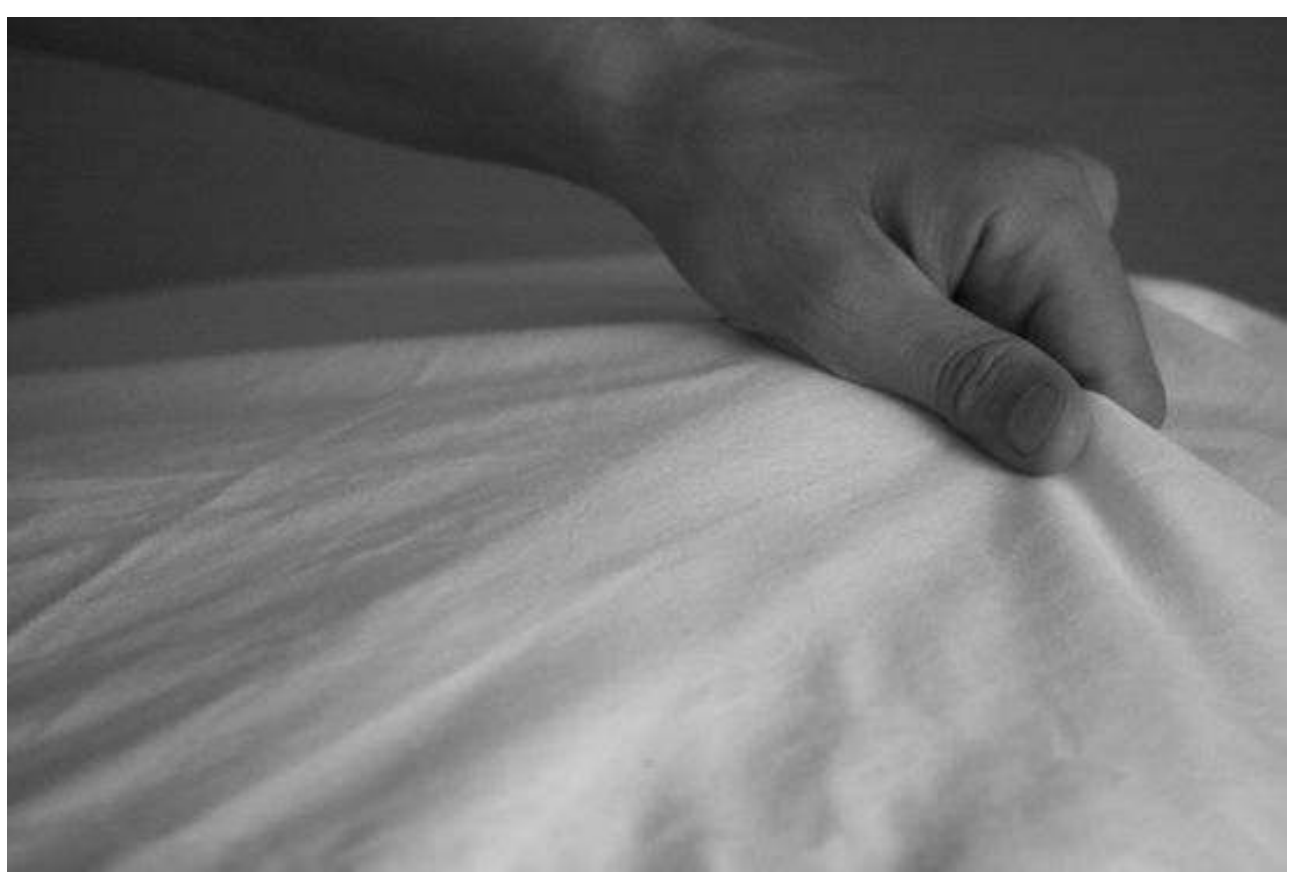

Ranger, défroisser, nettoyer, rendre présentable sans être représenté.

79 L'image de certains gestes exprime cette suppléance sans trace où les gestes effacent les défauts d'une dépendance liée au vieillissement et à une vie qui s'étiole et s'émiette peu à peu. L'image peut ainsi figurer des situations non représentées.

\section{Conclusion}

80 L'expérience photographique a été un tournant dans la recherche car, par la technique du plan serré, sont apparus des gestes inaperçus durant l'observation participante. Derrière l'appareil, le procédé de sensibilité photographique s'est mis au service du sujet de la recherche, à savoir la relation animée entre soignant et soigné. Cette expérience a permis de dépasser la confusion de l'immersion, et la production d'images a distancié la censure verbale de la quotidienneté, l'invisibilité des allants de soi et la banalité du vécu de l'accompagnement. Dans cette recherche, l'acte photographique m'a amené à conscientiser le geste de soin dans une concrétude singulière.

81 Cependant, cette focalisation circonscrit le geste au risque d'en exclure le contexte. La photographie nécessite une reconstruction fragmentaire de la réalité (Archimbaud, 2014). L'image seule ne peut se concevoir sans une conversion, une traduction ou un accompagnement à la perception. Semblable à la voix off du cinéma séquentiel de Jean Rouch, la photographie s'appréhende avec un complément délivrant le hors-cadre.

Dans le déroulement de l'enquête, cette situation expérientielle a pointé l'importance des négociations, des influences des malentendus et des impacts des vacances relationnelles. Embarquée dans une immersion de dix mois auprès des équipes du soin corporel, ce temps de "pause photographique » a révélé des écueils de négociation dans les attentes respectives d'une démarche de recherche. La production d'images nécessite une tractation délicate mais incontournable tant technique que relationnelle entre l'institution et l'enquêteur. 

l'invisible et l'indicible dictés par les logiques internes. La tension photographique comme productrice d'images a ravivé cette question du racontable et du regardable, détenus et prescrits par le domaine médical. Et si les relations avec les soignants du quotidien étaient restées cordiales, une atmosphère d'inquiétude persistait: celle de révéler des gestes impropres. Le personnel encadrant, quant à lui, restait vigilant et distant. Une exposition des photographies réalisées aurait entrainé des échanges supplémentaires, il ne voulait pas risquer un dérangement potentiel alors que ma présence au sein de l'établissement se finissait.

La complexité de l'usage de la photographie se retrouve également dans le passage d'échelle de réflexion. L'image produite peut se poser en médiatrice entre le micro et le macro, liant une relation privatisée et sensible aux actes techniques et politiques du soin. Cette problématique s'inscrit dans une mise en scène de l'intime (Tisseron, 2011) et s'approche d'une sensibilisation à l'interaction humaine dans le cadre de l'étique du care. Dans l'anthropologie du proche, la pratique photographique apparait une ouverture relationnelle dans laquelle l'image sert de point de convergence ou de divergence perceptuelle et fait sortir le quotidien du poids silencieux du banal.

De plus, de nombreuses situations n'ont pas fait déclencher l'appareil. L'outil photographique peut être soumis à la censure du photographe et devenir un frein pour l'enquête. Cependant qu'il y ait ou non image, la présence de l'appareil pose déjà un cadre qui invite à une nouvelle forme d'interaction riche en informations pour la recherche. Contrairement aux photographies journalistiques, ou « Le poids des mots le choc des images " (Sontag, 3003, 31), le chercheur-photographe (re)construit son terrain d'observation, au rythme de ses prises de vue. L'intention n'a pas été de l'ordre de la capture, mais plutôt sur le registre de la prise de conscience en images d'une rencontre au regard des gestes qui animent le quotidien des soignants et des soignés.

\section{BIBLIOGRAPHIE}

Archimbaud N. (2014), « Cerner l'approche globale du malade âgé en milieu hospitalier », Journal des anthropologues, Vol. 138, $n^{\circ} 3$, p. 221-238.

Bateson G. (1977,) « Les usages sociaux du corps à Bali », Actes de la recherche en sciences sociales, Vol. 14, n¹, p. 3-33.

Darmon M. (2005), « Le psychiatre, la sociologue et la boulangère : analyse d'un refus de terrain, Genèses, Vol. 58, n 1, p. 98-112.

Derbez B. (2010), « Négocier un terrain hospitalier », Genèses, Vol. 1, nº 78, p. 105-120.

Desmond M. (1979), Gestures, their origins and distribution, London, Jonathan Cape.

Desmond M. (1985), Magie du corps, Paris, Bernard Grasset.

Goffman E. (1974), Les rites d'interaction, Paris, Éditions de Minuit, Coll. « Le sens commun ».

Images du travail, travail des images, 4 | 2017 
Goffman E. (1991), Les cadres de l'expérience, Paris, Éditions de Minuit, Coll. « Le sens commun ».

Jehel P. J. (2000), « Photographie : sujet sensible. Un parcours comparatif parmi trois livres de photographies à caractère ethnologique », Journal des anthropologues, n 80-81, p. 161-191.

Piette A. (1992), « La photographie comme mode de connaissance anthropologique », Terrain, $\mathrm{n}^{\circ}$ 18, p. 129-136.

Piette A. (1996), Ethnographie de l'action : l'observation des détails, Paris, Métailié. Coll. « Leçons de choses ».

Sontag S. (2002), Devant la douleur des autres, Paris, Christian Bourgois.

Tisseron S. (2011), « Intimité et extimité », Communications, Vol. 88, n 1, p. 83-91.

\section{NOTES}

1. Certaines photos ont été rectifiées sous Lightroom4, logiciel de traitement photographique, les retouches portaient principalement sur la clarté par manque de lumière. Un léger recadrage a été parfois réalisé pour éviter notamment la reconnaissance des visages.

\section{RÉSUMÉS}

La photographie peut-elle être une source pour comprendre l'interaction entre soignant et soigné? Photographier les gestes de soin en maison de retraite est inhabituel et soulève la délicate question des intimités qui éloignent de tout regard l'aspect quotidien de l'acte de suppléance. Cet article invite à considérer l'acte photographique comme une méthode d'enquête ethnographique afin de (re)mobiliser la discussion autour d'une relation exclue à la verbalisation. La technique photographique se met ici en correspondance avec les gestes quotidiens afin de révéler au travers d'images de mains des modalités singulières d'interface. De cette démarche émergent des éléments supplémentaires à l'enquête immersive, afin de comprendre ce qui se fait et se vit.

Can photography be a source for understanding the interaction between caregiver and patient? Photographing the gestures of care in nursing homes is unusual and raises the delicate question of intimacies which take away the daily appearance of the act of substitution. This article invites us to consider the photographic act as a method of ethnographic inquiry in order to (re) mobilize the discussion around a relation excluded from verbalization. The photographic technique here corresponds to the daily gestures in order to reveal the singular interface modalities through images of hands. From this approach emerge additional elements to the immersive investigation, in order to understand what is being done and lived. 


\section{INDEX}

Keywords : images of hands, care relationships, interactions, retirement home, photographic notes, ethnographic approach

Mots-clés : images de mains, relations soignant-soigné, interactions, maison de retraite, notes photographiques, démarche ethnographique

\section{AUTEUR}

\section{DELPHINE MORAS}

Anthropologue de la santé et du vieillissement, formée aux psychopathologies du sujet âgé et à l'art-thérapie. Doctorante en Anthropologie et en Sociologie ED 483 ScSo, et membre du LADEC (Laboratoire d'Anthropologie Des Enjeux Contemporains), ses recherches se sont portées dans un premier temps sur les rituels funéraires en Béarn, pour se poursuivre sur les pratiques de soin en gérontologie.

Sa thèse : « l'Évidente Toilette ou le temps d'une relation, gestes de soin(s) face aux troubles de la maladie d'Alzheimer ", retrace une ethnographie en maison de retrait.

Elle a contribué à des projets institutionnels notamment :

- « Jardin de soin : parcours et rêverie » : création d'un jardin thérapeutique en Ehpad, avec JAS (Jardin, Art et Santé).

- «Au-delà du souvenir » : analyse de la demande et des besoins en accompagnement auprès des familles de personnes atteintes de troubles de la mémoire avec l'association France Alzheimer Rhône.

- «Au fil des temps » : ateliers de modelage en UHR auprès de personnes atteintes de la maladie d'Alzheimer. 